\title{
The role of asymmetries in coronal rain formation during thermal non-equilibrium cycles ${ }^{\star}$
}

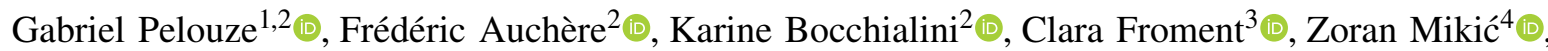 \\ Elie Soubrié ${ }^{2,5}$, and Alfred Voyeux ${ }^{2}$ \\ ${ }^{1}$ Centre for mathematical Plasma Astrophysics, Department of Mathematics, KU Leuven, Celestijnenlaan 200B bus 2400, \\ 3001 Leuven, Belgium \\ e-mail: gabriel.pelouze@ias.u-psud.fr \\ 2 Institut d'Astrophysique Spatiale, CNRS, Université Paris-Saclay, 91405 Orsay, France \\ ${ }^{3}$ LPC2E, CNRS/University of Orléans/CNES, 3A avenue de la Recherche Scientifique, Orléans, France \\ ${ }^{4}$ Predictive Science, Inc., San Diego, CA 92121, USA \\ 5 Institute of Applied Computing \& Community Code, Universitat de les Illes Balears, 07122 Palma de Mallorca, Spain
}

Received 2 February 2021 / Accepted 17 October 2021

\begin{abstract}
Context. Thermal non-equilibrium (TNE) produces several observables that can be used to constrain the spatial and temporal distribution of solar coronal heating. Its manifestations include prominence formation, coronal rain, and long-period intensity pulsations in coronal loops. The recent observation of abundant periodic coronal rain associated with intensity pulsations allowed for these two phenomena to be unified as the result of TNE condensation and evaporation cycles. On the other hand, many observed intensity pulsation events show little to no coronal rain formation.

Aims. Our goal is to understand why some TNE cycles produce such abundant coronal rain, while others produce little to no rain. Methods. We reconstructed the geometry of the periodic coronal rain event, using images from the Extreme Ultraviolet Imager (EUVI) onboard the Solar Terrestrial Relations Observatory (STEREO), and magnetograms from the Helioseismic and Magnetic Imager (HMI). We then performed 1D hydrodynamic simulations of this event for different heating parameters and variations of the loop geometry (9000 simulations in total). We compared the resulting behaviour to simulations of TNE cycles that do not produce coronal rain.

Results. Our simulations show that both prominences and TNE cycles (with and without coronal rain) can form within the same magnetic structure. We show that the formation of coronal rain during TNE cycles depends on the asymmetry of the loop and of the heating. Asymmetric loops are overall less likely to produce coronal rain, regardless of the heating. In symmetric loops, coronal rain forms when the heating is also symmetric. In asymmetric loops, rain forms only when the heating compensates for the asymmetry.
\end{abstract}

Key words. Sun: atmosphere - Sun: corona - Sun: oscillations - Sun: UV radiation

\section{Introduction}

Understanding the heating of coronal loops has been a longstanding problem in solar physics. Numerous physical mechanisms have been proposed (see Cranmer \& Winebarger 2019, and references therein). The main challenge is to identify which ones are predominant in the solar atmosphere. This requires strong observational diagnostics to discriminate between candidate mechanisms. To that end, it is important to constrain the spatial and temporal properties of the heating.

Thermal non-equilibrium (TNE) provides several valuable observables to constrain coronal heating. In the solar corona, TNE occurs in coronal loops which are subject to a highly stratified heating localised close to the surface (Antiochos \& Klimchuk 1991; Antiochos et al. 1999, 2000; Karpen et al. 2001; Klimchuk \& Luna 2019; Klimchuk 2019; Antolin 2020). In this configuration, the heating may not balance the radiative losses at the apex of the loop. Such loops thus have no thermal equilibrium state. As a result of the footpoint heating, chromospheric plasma evaporates, and upflows develop in both legs of the loop. Plasma accumulates at the

\footnotetext{
$\star$ Movie associated to Fig. 5 is available at https:// www . aanda.org
}

loop apex, leading to an increase in the density. The radiative losses increase quadratically with the density, which at some point results in an inevitable cooling since the heat input is constant. As the temperature drops, the radiative losses further increase (Pottasch 1965; McWhirter et al. 1975), and the plasma quickly starts to condense close to the apex of the loop. Depending on the geometry of the magnetic field, the condensation may accumulate at coronal heights and form a prominence (e.g., Mok et al. 1990; Antiochos \& Klimchuk 1991; Antiochos et al. 1999, 2000; Karpen et al. 2001, 2005; Xia et al. 2011, 2014; Xia \& Keppens 2016). Alternatively, the condensation may be evacuated from the loop and fall towards one of its footpoints. In this latter case, the process starts over, resulting in condensation and evaporation cycles, also referred to as TNE cycles (Kuin \& Martens 1982; Martens \& Kuin 1983; Craig \& Schulkes 1985). The characteristics of TNE cycles (e.g., its period, or the minimum and maximum temperature) depend on the spatial and temporal properties of the heating, as well as the geometry of the loop. These cycles thus constitute a valuable tool to constrain the heating of coronal loops.

Thermal non-equilibrium cycles result in long-period (2 to $16 \mathrm{~h}$ ) pulsations of the extreme-ultraviolet (EUV) emission of coronal loops. Such pulsations were first detected by 
Auchère et al. (2014) in images from the Extreme-ultraviolet Imaging Telescope (EIT; Delaboudinière et al. 1995) onboard the Solar and Heliospheric Observatory (SOHO; Domingo et al. 1995), and by Froment etal. (2015) in images from the Atmospheric Imaging Assembly (AIA; Lemen et al. 2012; Boerner et al. 2012) onboard the Solar Dynamics Observatory (SDO; Pesnell et al. 2012). These long-period intensity pulsations have been found to be very common in coronal loops (Auchère et al. 2014; Froment 2016). This provides a strong indication that a subset of coronal loops are subject to a stratified heating, localised close to their footpoints.

Another key consequence of TNE is the formation of coronal rain. It occurs when plasma at coronal heights condenses to chromospheric temperatures before being evacuated (Schrijver 2001; Müller et al. 2003，2004，2005; De Groof et al. 2005; Antolin et al. 2010). This condensed plasma falls along the coronal loop, forming blob-like structures that are observed in chromospheric and transition-region lines (Kawaguchi 1970; Leroy 1972; Foukal 1978; Schrijver 2001; O'Shea et al. 2007; De Groof et al. 2004, 2005; Antolin et al. 2010, 2012; Antolin \& Rouppe van der Voort 2012; Vashalomidze et al. 2015). Coronal rain can also occur in post-flare loops as a result of the intense transient heating from the flare (Scullion et al. 2016). While the formation of periodic coronal rain during TNE cycles had long been suspected (e.g., Antolin et al. 2010), it proved challenging to observe.

The first case of periodic coronal rain associated with EUV intensity pulsations was reported by Auchère et al. (2018). This event, nicknamed the 'rain bow', was observed in long transequatorial loops observed off-limb by SDO/AIA. The authors report the detection of EUV intensity pulsations from plasma at coronal temperatures, accompanied by periodic coronal rain observed in the $304 \AA$ channel. The observation in the $304 \AA$ channel indicates that the condensations cool down to at least $90000 \mathrm{~K}$ during the TNE cycle. The rain is also seen in images of the $\mathrm{H} \alpha$ coronograph of the Pic du Midi observatory (Koechlin et al. 2019), bringing the temperature range all the way down to the chromospheric level (this was not published by Auchère et al. 2018). Finally, at the end of the observation sequence, the coronal rain remains longer at the apex of the loop before falling, looking similar to the onset formation of a prominence. This event confirms that coronal rain and EUV intensity pulsations are indeed two aspects of TNE cycles. It also hints to the fact that prominences could result from extremely similar heating or magnetic field configurations. Further observations of coronal rain associated with EUV intensity pulsations were performed by Froment et al. (2020).

On the other hand, TNE cycles can occur with little to no formation of coronal rain. This happens when the condensing plasma is reheated before it has had time to cool down to chromospheric temperatures. Entirely ruling out the presence of coronal rain in observations of TNE cycles is difficult because the finer coronal rain drops may not be resolved by the available instruments. However, Froment et al. (2015) performed a detailed analysis of the thermal evolution of three EUV intensity pulsation events, and they concluded that some of the observed cycles were unlikely to produce coronal rain. This was later confirmed by the analysis of off-disk observation of these events by the Extreme Ultraviolet Imager (EUVI; Wuelser et al. 2004) from the Sun Earth Connection Coronal and Heliospheric Investigation instrument suite (SECCHI; Howard et al. 2008) onboard the Solar Terrestrial Relations Observatory (STEREO; Driesman et al. 2008; Kaiser et al. 2008). Finally, flows develop during all TNE cycles, even if the plasma remains at coronal temperatures throughout the cycle and no condensation forms
(Mikić et al. 2013). The observation of such flows of plasma at coronal temperature was reported by Pelouze et al. (2020).

The different consequences of TNE in coronal loops have also been investigated in a number of simulation studies. The first simulation studies of TNE focused on the evolution of plasma inside a fixed coronal loop, as a response to an imposed heating. A common approach is to reduce a 3D magnetohydrodynamic (MHD) problem to a 1D hydrodynamic problem, where the plasma evolves along a fixed magnetic field line. Such simulations produced both TNE cycles with coronal rain (Kuin \& Martens 1982; Martens \& Kuin 1983; Karpen et al. 2001, 2005; Müller et al. 2003, 2004, 2005; Antolin et al. 2010; Xia et al. 2011) and prominences (Antiochos \& Klimchuk 1991; Antiochos et al. 1999, 2000; Karpen et al. 2001, 2005, 2006; Karpen \& Antiochos 2008; Xia et al. 2011). However, simulations by Mikić et al. (2013) show that the characteristics of TNE cycles depend more strongly on the geometry of the loop than assumed in previous works. In particular, they demonstrate that TNE cycles without coronal rain (incomplete condensations) could develop in loops with a non-uniform cross-sectional area. Froment et al. (2017) later performed simulations that accurately reproduced the TNE cycles with little coronal rain observed by Froment et al. (2015). Froment et al. (2018) then investigated how the heating and the geometry of the loop interact to produce TNE cycles. To that end, they simulated the response of three loops of different geometries to a range of heating functions. They conclude that TNE cycles only occur for a fraction of the explored heating parameters, and that they require a specific match between the loop geometry and the heating function.

However, it is still unclear why some TNE cycles produce little to no coronal rain (e.g., Froment et al. 2015), while some produce abundant coronal rain (e.g., the rain bow, Auchère et al. 2018). Furthermore, the rain bow forms very abundant rain showers compared to the other periodic coronal rain event (Froment et al. 2020), and the observations even suggests the onset formation of a prominence. Froment et al. (2018) have shown that cycles with and without coronal rain can occur within the same loop, given different heating functions. Our goal is to better understand which geometry and heating parameters lead to the formation of coronal rain during TNE cycles, and to the extreme behaviour of the rain bow. To that end, we performed numerical simulations of the rain bow event (Auchère et al. 2018). We derived eight variants of the geometry and studied how the plasma evolves in each loop as a response to a large number of heating functions. We then compared the results to the parametric study realised by Froment et al. (2018) for different loop geometries, in particular that of a loop experiencing TNE cycles with little coronal rain. This means comparing an event with abundant periodic coronal rain forming in transequatorial loops (the rain bow), to TNE cycles with little to no rain occurring in long loops located at the edge of an active region (Froment et al. 2018, loop B).

We first determine the geometry of the rain bow, which is required to perform the simulations (Sect. 2). We then present the numerical set-up (Sect. 3.1), and the exploration of the geometries and heating parameter space (Sect. 3.2). This parametric study shows that all evolution scenarios of TNE (pulsation at coronal temperatures, periodic coronal rain, and prominence formation) can occur in the rain bow loop, depending on the heating function (Sect. 3.3). Additionally, we estimate the spatial distribution of the heating in the observed loops (Sect. 3.4). We then compare our simulations to the parametric study performed by Froment et al. (2018) for different loop geometries in order to understand how the asymmetry of the loop and the heating result 
(a) SDO

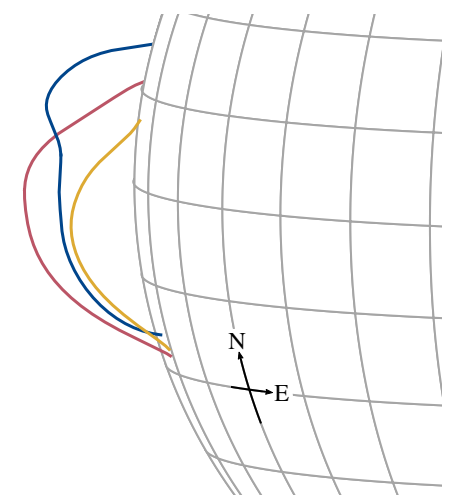

(b) STEREO B

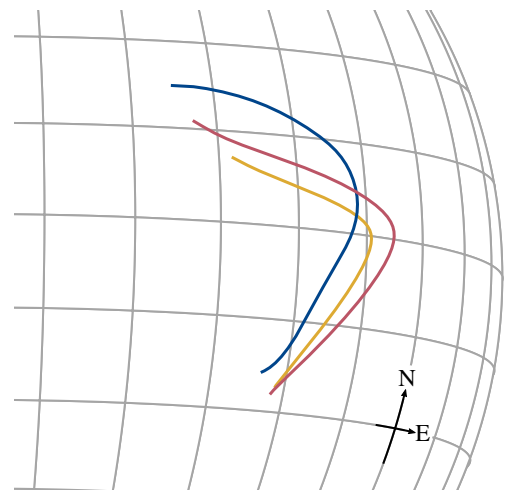

(c) South

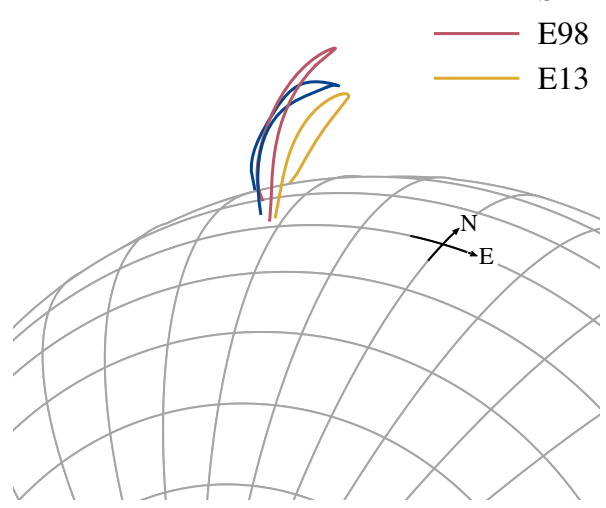

Fig. 1. Geometries of the rain bow from the stereoscopic reconstruction (S) and from the magnetic field extrapolation (E13 and E98), viewed from the position of SDO (a), STEREO B (b), and near the solar south pole (c). The heliographic grid is spaced by $10^{\circ}$. The cross indicating the north and east directions on the solar sphere is located at the latitude of $-20^{\circ}$ and the Carrington longitude of $220^{\circ}$.

in the formation of coronal rain during TNE cycles (Sect. 4). Finally, we summarise our conclusions in Sect. 5.

\section{Geometry of the rain bow}

To perform numerical simulations of the rain bow event (Auchère et al. 2018), it is necessary to know the 3D geometry of the loop. For loops observed on-disk, the geometry can be reconstructed using a magnetic field extrapolation (e.g., Mikić et al. 2013; Froment et al. 2017). However, this is not possible for loops observed off-limb such as the rain bow as there exist no cotemporal magnetogram. The closest magnetograms suitable for extrapolation are necessarily out of date, having been taken when the loop was observed on-disk from Earth. In the case of the rain bow, which is observed at the east limb, such magnetograms were recorded either at the previous solar rotation (about 20 days before), or once the structure reached the disk centre (about six days later).

However, the rain bow was also observed on-disk by SECCHI/EUVI onboard STEREO B. We combined these observations with images from SDO/AIA to get a stereoscopic view of the loop and to reconstruct its geometry (Sect. 2.1 and Appendix A). Then, we used synoptic magnetograms from the Helioseismic and Magnetic Imager (HMI; Scherrer et al. 2012) onboard SDO to get an estimation of the magnetic field expansion in the loop (Sect. 2.2 and Appendix B).

\subsection{Stereoscopic reconstruction}

We used stereoscopic observations of SDO/AIA (near Earth) and STEREO B/SECCHI/EUVI (separation of $115^{\circ}$ with Earth) to reconstruct the tri-dimensional geometry of the rain bow loop. The reconstruction is summarised in Appendix A. The resulting 3D loop shape is shown in Fig. 1 (line S). It has a length of $635.36 \mathrm{Mm}$ and is oriented in the north-south direction. While it appears to be in a plane when seen from SDO (Fig. 1a), the loop is clearly non-planar and leans towards the east of the solar sphere. As a result, it is much longer than it appears to be in AIA images (Auchère et al. 2018 report a length of $438 \mathrm{Mm}$ in the plane of the sky). Figures $2 \mathrm{a}$ and $\mathrm{b}$ show the altitude $z$ and the projected gravity $g_{\|}$as a function of the position along the loop $s$ divided by the loop length $L$ ( $s=0$ corresponds to the north footpoint of the loop). These plots reveal the presence of a dip near the apex, which is an unusual feature for a coronal loop. The dip has a depth of $2 \mathrm{Mm}$ and a width of $53 \mathrm{Mm}$. The loop legs are otherwise rather symmetric, both having comparable altitude profiles. In addition, both footpoints are almost vertical, with a projected gravity equal to the solar surface gravity.

The stereoscopic reconstruction has a few limitations. The first one is the that loop footpoints are not well constrained. The second limitation is that the geometry was only reconstructed at a given time and, therefore, it does not reflect the possible time evolution of the magnetic field. Finally, it only provides us with the shape of the loop, but not its cross-sectional area.

\subsection{Magnetic field extrapolation}

The hydrodynamic simulations also need the relative crosssectional loop area as input. The relative loop area $A$ is inversely proportional to the magnetic field strength $B: A(s)=B(0) / B(s)$. We normalised the cross-sectional area to the area of the north footpoint (i.e. $A(0)=1$ ). To estimate the loop expansion of the rain bow, we performed a potential magnetic field extrapolation from a low-resolution synoptic line-of-sight magnetogram constructed using SDO/HMI data. The construction of the synoptic magnetogram and the magnetic extrapolation are detailed in Appendix B.

We selected two magnetic field lines extrapolated from this magnetogram. The shape of these lines is shown in Fig. 1 (lines E13 and E98). The altitude, projected gravity, and relative loop area are shown in Fig. 2. Both loops are non-planar and inclined towards the east (albeit less than the stereoscopically reconstructed shape S), and they have no apex dip. Line E98 has a length of $628.86 \mathrm{Mm}$, and it is overall similar to the stereoscopic shape $S$ with no apex dip. The dip present in shape $S$ could result from the deformation of the magnetic field by the abundant coronal rain because the stereoscopic reconstruction was performed late in the observing sequence, after a lot of rain had already formed. Such deformation would not be visible in the result of the potential extrapolation. Line E13 is shorter, with a length of 465.54 Mm.

As discussed in the appendix, the potential extrapolation yields a very coarse estimation of the magnetic field. To mitigate this, we built six cross-sectional area profiles from four extrapolated magnetic field lines, and we combined them with the shape from the stereoscopic reconstruction $\mathrm{S}$. Thus, we can later verify whether the simulation results strongly depend on the loop area expansion and assess the importance of the uncertainties 
(a) Altitude

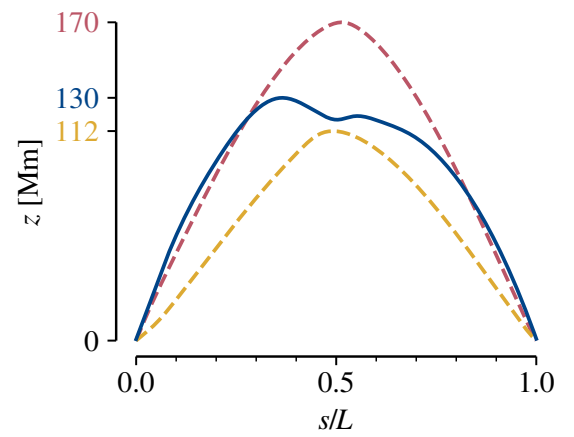

(b) Projected gravity

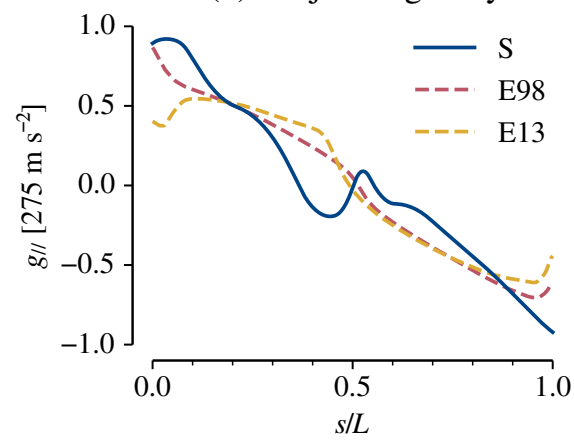

(c) Relative loop area

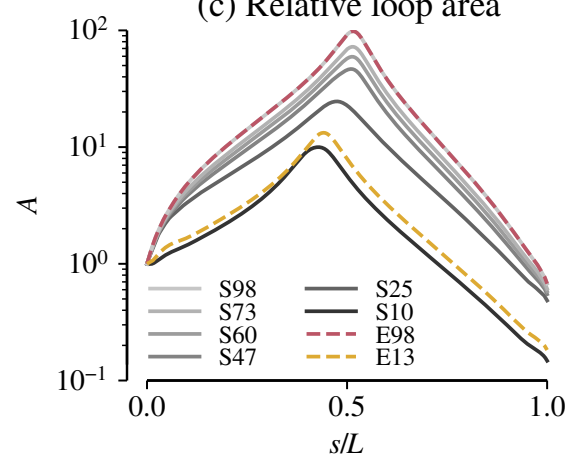

Fig. 2. Altitude (a), projected gravity (b), and relative loop cross-sectional area (c) as a function of the position along the loop $s$ divided by the loop length $L$, for the geometries shown in Fig. 1, and the six loop area profiles generated for the $\mathrm{S}$ geometry. The maximum values of the altitude and relative loop area are marked on the corresponding $y$-axes. The position $s=0$ corresponds to the north footpoint of each loop.

associated with the extrapolation. We labelled the resulting geometries with their maximum relative area expansion: $\mathrm{S} 10$, S25, S47, S60, S73, and S98. The cross-sectional area profiles of these geometries are shown in Fig. 2c. We performed numerical simulations for the six geometries using the stereoscopic loop shape, as well as for the two extrapolated geometries.

\section{Numerical simulations of TNE cycles}

\subsection{Numerical set-up}

We use the 1D hydrodynamic set-up described by Mikić et al. (2013). This set-up allows the evolution of the plasma inside a fixed magnetic field tube with a non-uniform cross-sectional area to be computed. To that end, the equations for the conservation of mass, momentum, and energy are solved. The energy equation includes radiative losses, thermal conduction, and a constant, non-uniform imposed heating term $H(s)$. The radiative losses were computed using the Chianti atomic database (version 7.1; Dere et al. 1997; Landi et al. 2013), coronal abundances (Feldman et al. 1992; Feldman 1992; Grevesse \& Sauval 1998; Landi et al. 2002), and the Chianti collisional ionisation equilibrium model (Dere et al. 2009). The thermal conduction was computed using the Spitzer conductivity $\kappa_{\|}(T)$ (Spitzer 1962) above the cut-off temperature $T_{c}=250000 \mathrm{~K}$, and a constant conductivity $\kappa_{\|}\left(T_{c}\right)$ at lower temperatures (Lionello et al. 2009; Mikić et al. 2013). This artificially broadens the transition region, allowing for larger numerical grid cells and thus a significant reduction in the computation time. Mikić et al. (2013) show that the use of the modified conductivity, as well as the choice of the radiative loss function and of the abundances, do not significantly change the simulation results. In this set-up, it is assumed that the plasma is composed of fully ionised hydrogen, and thus that the electron and ion number densities are equal). Furthermore, the ion and electron temperatures are assumed to be equal.

The loop was initialised in hydrostatic equilibrium, with a parabolic temperature profile reaching $2 \mathrm{MK}$ at the loop midpoint, and a $3.5 \mathrm{Mm}$-thick chromosphere at a constant temperature of $20000 \mathrm{~K}$. Throughout the simulation, the constant chromospheric temperature $T_{\text {ch }}$ and number density $N_{\text {ch }}$ were imposed at the domain boundaries $(s=0$ and $s=L)$. We set $T_{\mathrm{ch}}=20000 \mathrm{~K}$ and $N_{\mathrm{ch}}=3 \times 10^{19} \mathrm{~m}^{-3}$. This boundary condition allowed us to maintain a layer of chromospheric plasma at both footpoints, which served as a mass reservoir for evaporation and absorbed falling condensations. Previous studies using this numerical set-up imposed $N_{\mathrm{ch}}=6 \times 10^{18} \mathrm{~m}^{-3}$ (Mikić et al.
2013; Downs et al. 2016; Froment et al. 2017, 2018). However, some simulations of the rain bow produce very large condensations that are not absorbed by the resulting chromospheric layer. Such condensations cause numerical issues that interrupt the simulation when they reach the boundaries of the computation domain. To reduce the number of interrupted simulations, we increased the chromospheric density to $3 \times 10^{19} \mathrm{~m}^{-3}$, thus increasing the thickness of the chromospheric layer. While this value may be larger than typical chromospheric densities, it does not affect the solution in the coronal part of the loop (see discussion in Mok et al. 2005). We verified that the behaviour in the coronal part of the simulation did not change when using $N_{\mathrm{ch}}=6 \times 10^{18} \mathrm{~m}^{-3}$ and $3 \times 10^{19} \mathrm{~m}^{-3}$.

The loop is subject to a constant stratified heating, localised near both footpoints. The heating consists of a uniform background term, and two exponentially decreasing terms, one for each leg of the loop. It is given by

$H(s)=H_{0}+H_{1}\left(\mathrm{e}^{-g(s) / \lambda_{1}}+\mathrm{e}^{-g(L-s) / \lambda_{2}}\right)$,

where $H_{0}$ is the constant background volumetric heating rate, $H_{1}$ the stratified volumetric heating rate, and $\lambda_{1}$ and $\lambda_{2}$ are the scale heights of the stratified heating at both footpoints. The term $g(s)=\max (s-\Delta, 0)$ allows for constant heating in the chromosphere, up to $\Delta=5 \mathrm{Mm}$ away from both footpoints. To obtain a stratified heating, we chose $H_{1} \gg H_{0}$ and $\left\{\lambda_{1}, \lambda_{2}\right\}<L$. A greater stratification is obtained with smaller heating scale height values, or higher values of $H_{1} / H_{0}$. We note that more energy is deposited in the loop as either $\lambda_{1}$ or $\lambda_{2}$ increased. A symmetric heating is obtained when $\lambda_{1}=\lambda_{2}$.

We used 10000 grid points along the loop, with variable cell sizes of $4 \mathrm{~km}$ in the chromosphere, $40 \mathrm{~km}$ in the transition region, and $400 \mathrm{~km}$ around the loop apex. We let the system evolve for $72 \mathrm{~h}$.

\subsection{Parameter-space scan: Loop geometry and heating}

We investigated how the evolution of the plasma in the loop depends on the parameters of the heating function (Eq. (1)), the cross-sectional area of the loop, and the presence of a dip at the apex of the loop. To that end, we performed simulations for the eight geometries described in Sect. 2 and shown in Figs. 1 and 2. Geometries S10, S25, S47, S60, S73, and S98 share the stereoscopically reconstructed loop shape, with a length of $635.36 \mathrm{Mm}$ and a dip at the apex. Their maximum area expansions vary from 10 to 98 . Geometries E13 and E98 were obtained from magnetic extrapolations and they do not have apex dips. Geometry E98 
Table 1. Explored parameter space.

\begin{tabular}{lccccc}
\hline \hline Geometry & $\begin{array}{c}L \\
{[\mathrm{Mm}]}\end{array}$ & $A_{\max }$ & $\begin{array}{c}H_{0} \\
{\left[\mathrm{nW} \mathrm{m}^{-3}\right]}\end{array}$ & $H_{1} / H_{0}$ & $\left\{\lambda_{1}, \lambda_{2}\right\} / L$ \\
\hline S10 & & 10.0 & 20.3 & Five & \\
S25 & & 24.6 & 19.0 & values & \\
S47 & 635.36 & 46.7 & 15.5 & each: & 15 values \\
S60 & & 59.5 & 14.5 & 1280, & each, \\
S73 & & 72.5 & 14.0 & 2560, & from $1 \%$ \\
S98 & & 98.4 & 13.0 & 5120, & to $20 \%$. \\
E98 & 628.86 & 98.4 & 14.0 & 10240, & \\
E13 & 465.54 & 13.3 & 53.0 & 20480. & \\
\hline
\end{tabular}

Notes. Columns are: Simulated geometry name, loop length $L$, maximum relative area expansion $A_{\max }$, constant background heating $H_{0}$, explored values of the stratified heating intensity $H_{1}$, and explored heating scale heights $\lambda_{1}$ and $\lambda_{2}$.

is the closest to the stereoscopic geometries, with a length of $628.86 \mathrm{Mm}$. E13 is shorter, with a length of $465.54 \mathrm{Mm}$.

For each of these geometries, we chose $H_{0}$ such that the apex temperature is approximately $1 \mathrm{MK}$ when the loop is subject to uniform heating (i.e. $H_{1}=0$ ). We then chose five values for $H_{1}$, expressed as multiples of $H_{0}$ : $(1280,2560,5120,10240,20480) \times H_{0}$. We also selected 15 values for each heating scale height $\lambda_{1}$ and $\lambda_{2}$, ranging from $1 \%$ to $20 \%$ of the loop length $L$. While the most extreme heating functions may no longer correspond to realistic coronal conditions (typically functions with high values of all three parameters), this choice of parameters allowed us to not neglect some realistic functions where only one or two parameters reached extreme values. In particular, we show in Sect. 3.4 that a high value of $H_{1} / H_{0}$ is required in order to reproduce the observed behaviour of the rain bow.

We performed simulations for all possible combinations of these heating parameters. This corresponds to 1125 simulations for each loop geometry, that is a total of 9000 simulations. The properties of the loop geometries and of the explored heating parameters are summarised in Table 1.

\subsection{Results: All TNE consequences in the same simulation set}

For each simulation, we obtained the temperature $T$, electron number density $N_{\mathrm{e}}$, pressure $p$, and velocity along the loop $v_{\|}$as a function of the position along the loop and time. In Fig. 3a, we show the evolution of the simulations performed with the geometry S47, a heating rate of $H_{1}=2560 H_{0}=39.7 \mu \mathrm{W} \mathrm{m}^{-3}$, and all explored values of the heating scale heights $\lambda_{1}$ and $\lambda_{2}$. This corresponds to 225 of the total 9000 simulations. Different TNE behaviours were obtained depending on the values of $\lambda_{1}$ and $\lambda_{2}$ :

- TNE cycles without formation of coronal rain, where the plasma in the coronal part of the loop remains at coronal temperatures throughout the simulation (Fig. 3b);

- TNE cycles with formation of coronal rain (Fig. 3c);

- the formation of a prominence-like structure, where condensed $^{1}$ plasma remains trapped in the dip at the apex of the loop (Fig. 3d);

\footnotetext{
1 The temperature and electron-number density reach chromospheric values, typically $T \sim 0.1 \mathrm{MK}$ and $N_{\mathrm{e}} \sim 10^{16} \mathrm{~m}^{-3}$.
}

- finally, a few cases with no TNE where the loop reaches a steady state, that is either a static equilibrium or continuous siphon flows (e.g., at $\lambda_{1}=6.35 \mathrm{Mm}, \lambda_{2}=127 \mathrm{Mm}$ ).

The cycles without coronal rain correspond to the cycles with incomplete condensations (IC) described by Mikić et al. (2013) and Froment et al. (2018). Cycles with coronal rain correspond to cycles with complete condensations (CC).

The beginning of simulations forming a prominence-like structure is very similar to that of simulations showing TNE cycles with coronal rain. Both begin with an initial relaxation, followed by a plasma condensation. However, in the case of the prominence-like structures, the condensation remains trapped in the dip at the apex of the loop. In the case of TNE cycles with coronal rain, the condensation is free to fall along one of the loop legs.

These simulations show the three behaviours that can result from TNE in coronal loops: evaporation and condensation cycles with coronal rain, cycles without coronal rain, and the formation of a prominence-like structure. As the examples in Fig. 3 show, different events can occur within the same loop geometry, and with similar heating parameters. Simulations by Mikić et al. (2013), Mok et al. (2016), and Froment et al. (2018) have already shown that cycles with and without coronal rain can occur in the same loop. However, the current parametric study is, to the best of our knowledge, the first to show that the three consequences of TNE can occur within the same loop geometry.

\subsubsection{Analysis of simulation results}

To reduce the large number of simulations, we summarise each simulation to a few characteristic quantities shown in Fig. 4. The maximum temperature and electron number density reached around the apex of the loop $\left(z>0.9 z_{\max }\right)$ during the second half of the simulation $(t>36 \mathrm{~h})$ are shown in Figs. $4 \mathrm{a}$ and b, respectively. The velocity averaged at the loop apex during the second half of the simulation is shown in Fig. 4c. Finally, the type of behaviour obtained in each simulation is presented in Fig. 4d.

Determining the behaviour in each simulation first requires identifying simulations with TNE cycles, and then those which produce coronal rain. It also requires one to identify the simulations that form prominence-like structures. Froment et al. (2018) detected simulations with pulsations in the Fourier space. However, many of the cycles produced in our simulations have very long periods, ranging from 20 to $30 \mathrm{~h}$. This gives between 2.5 and 3.5 periods in a simulation of $72 \mathrm{~h}$, which is not easily detected in the Fourier space. We thus identified pulsations manually by looking at the evolution of the temperature in the loops. We then applied the same criterion as Froment et al. (2018) to detect coronal rain: a simulation was considered to produce coronal rain if the temperature in the coronal part of the loop (defined as $z>20 \mathrm{Mm}$ ) locally dropped below $0.5 \mathrm{MK}$ after the first $10 \mathrm{~h}$ of the simulation. Similarly, prominence-like structures were identified manually based on the temperature evolution.

\subsubsection{Stereoscopically reconstructed loop}

We first present the result of the simulations performed with the geometries S10 to S98. These geometries all use the stereoscopically reconstructed loop shape, combined with different crosssectional area profiles.

The event classification for all simulations is shown in Fig. 4d. TNE occurs in a large fraction of the explored parameter space, in particular for smaller values of $H_{1} / H_{0}$. This includes both TNE cycles (with and without coronal rain) and the formation of prominence-like structures. Prominence-like structures 


\section{(a) Overview}
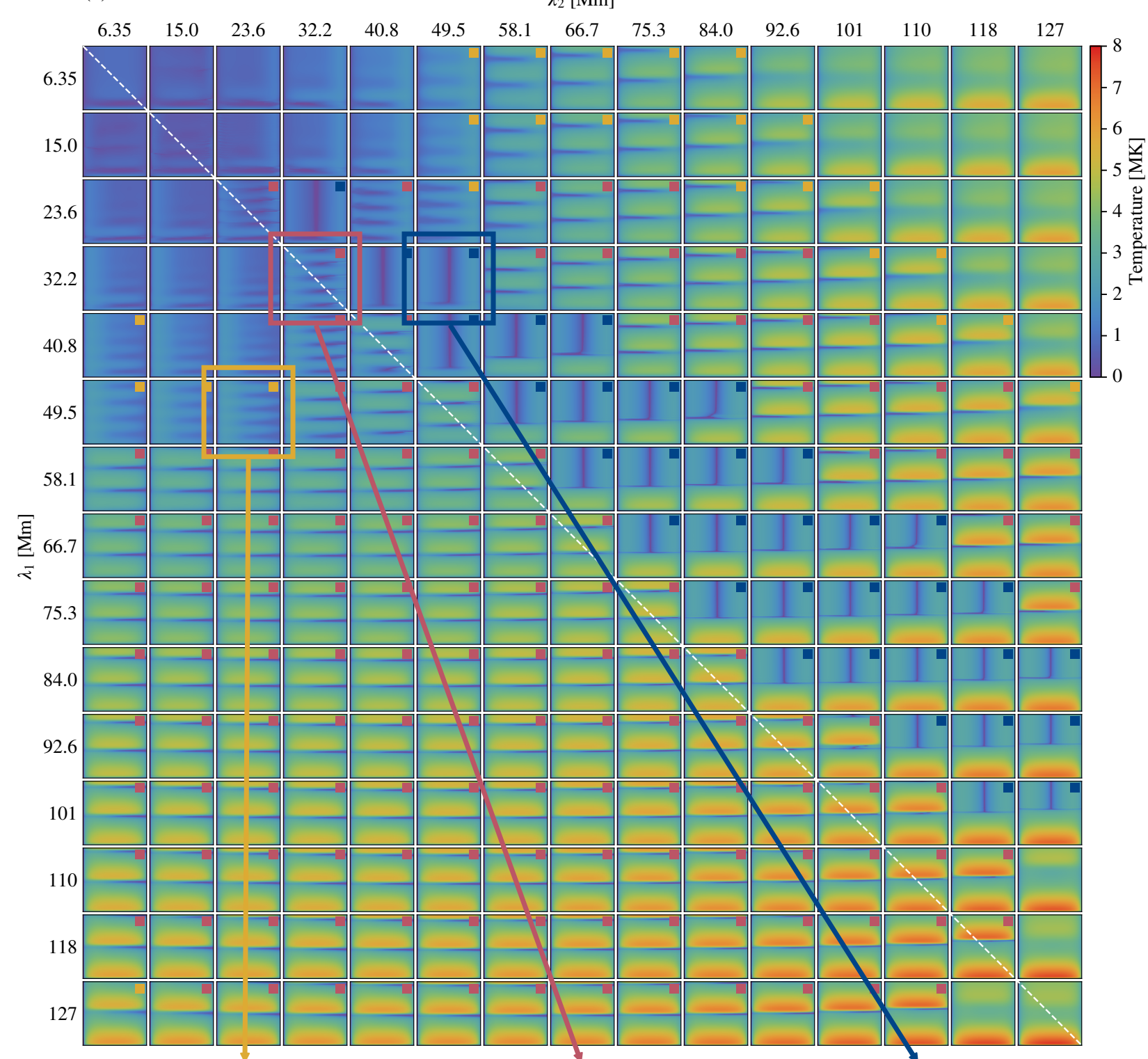

(b) TNE cycle with no coronal rain

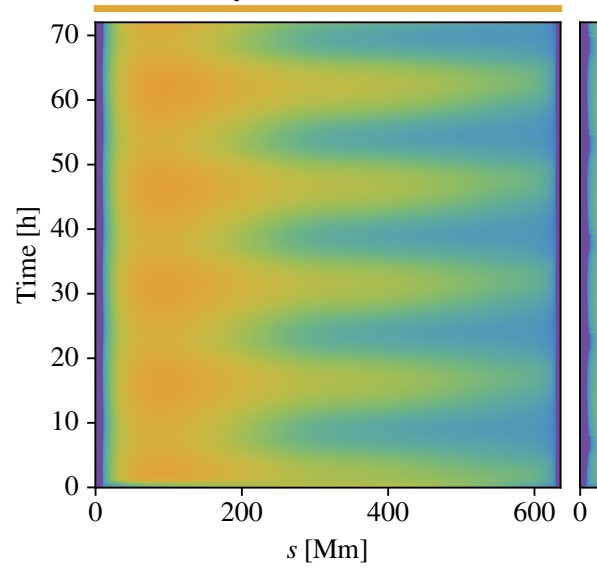

(c) TNE cycle with coronal rain

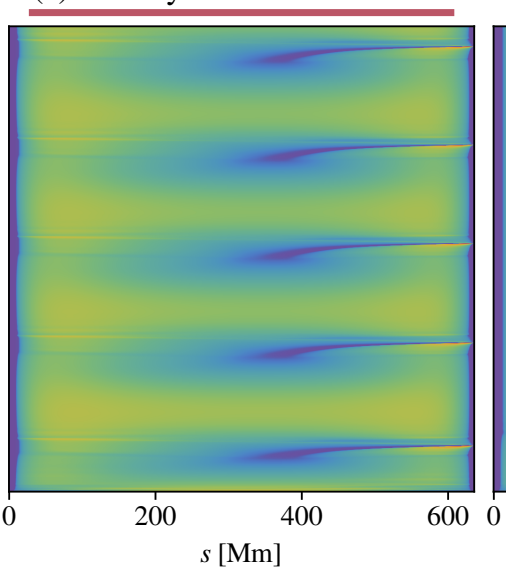

(d) Prominence

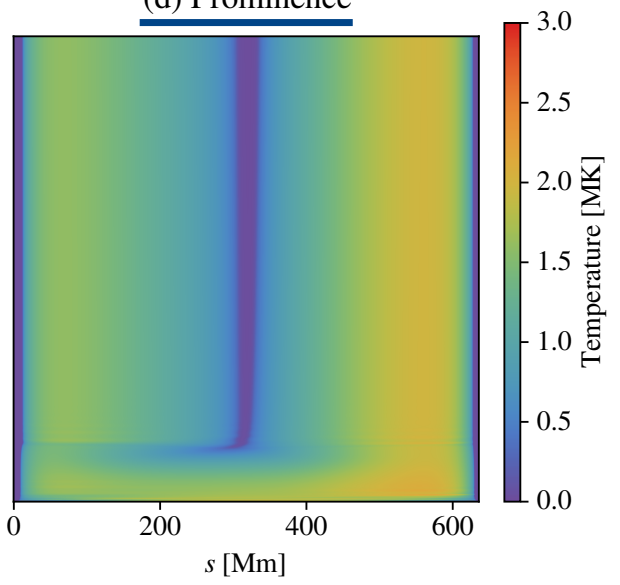

Fig. 3. Temperature evolution for the 225 simulations of the rain bow using the geometry S47 (stereoscopically reconstructed shape, with a maximum area expansion $A_{\max }=47$ ), a constant heating rate $H_{0}=15.5 \mu \mathrm{W} \mathrm{m}{ }^{-3}$, and a stratified heating rate $H_{1}=2560 H_{0}=39.7 \mu \mathrm{W} \mathrm{m}{ }^{-3}$. (a) Overview of the simulations performed with $\lambda_{1}$ and $\lambda_{2}$ each varying from $1 \%$ to $20 \%$ of the loop length $L$. Each sub-plot shows the evolution of temperature as a function of the position along the loop $s$ ( $x$-axis, ranging from 0 to $L=635.36 \mathrm{Mm}$ ) and of time ( $y$-axis, ranging from 0 to $72 \mathrm{~h}$ ). Event types are marked by coloured squares: TNE cycles with no coronal rain ( $\square$ ), cycles with rain ( $\square$ ), and prominence-like structures ( $\square$ ). The white dashed line corresponds to $\lambda_{1}=\lambda_{2}$. (b) Sample simulation showing TNE cycles without coronal rain. (c) Sample simulation showing TNE cycles with coronal rain. (d) Sample simulation showing the formation of a prominence-like structure. 
(a) Maximum apex temperature $[\mathrm{MK}]$

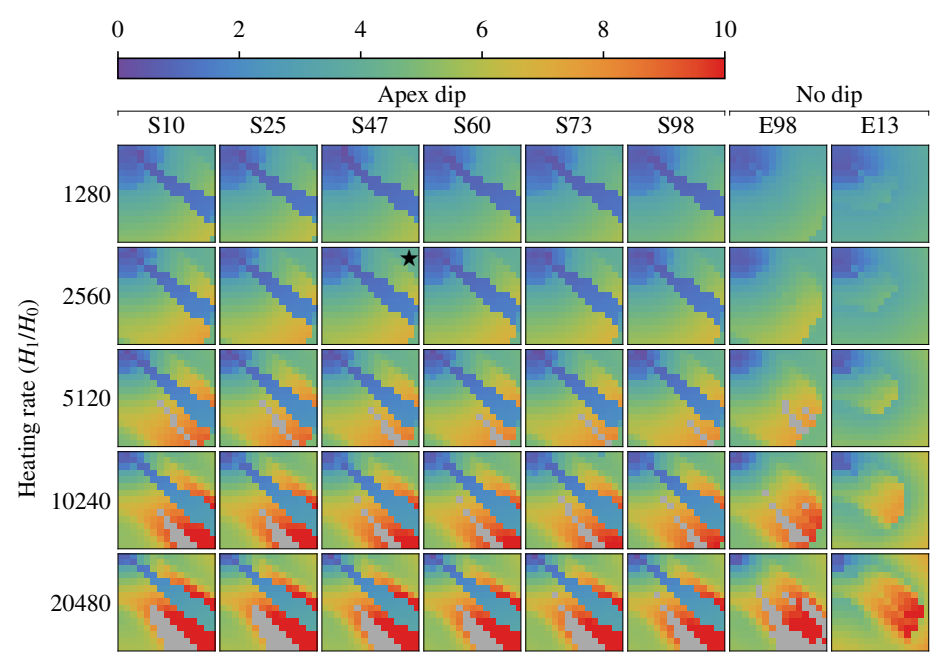

(c) Average apex velocity $\left[\mathrm{km} \mathrm{s}^{-1}\right]$

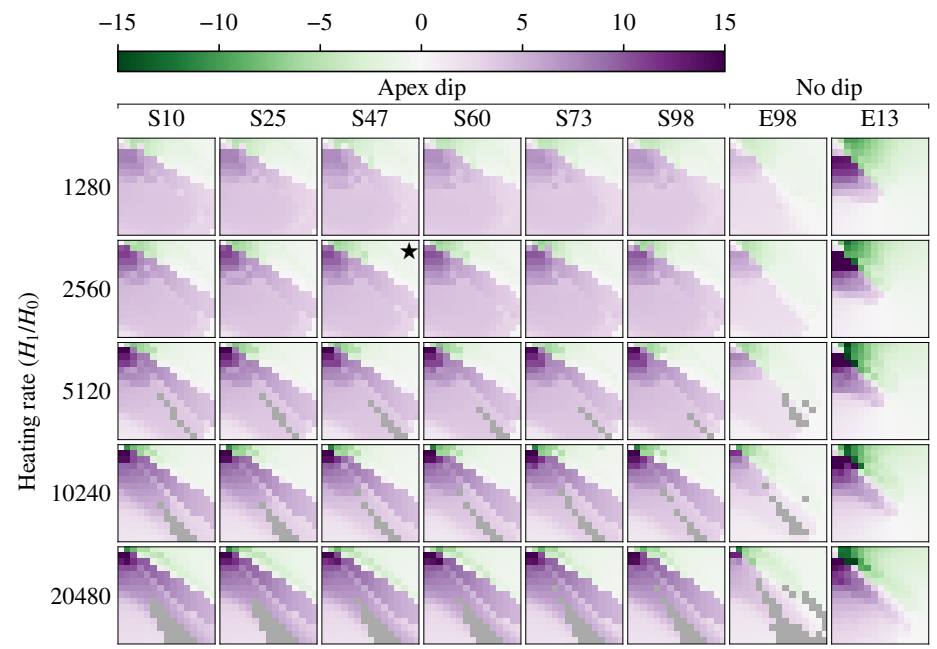

(b) Maximum apex electron number density $\left[\mathrm{m}^{-3}\right]$ $10^{14}$

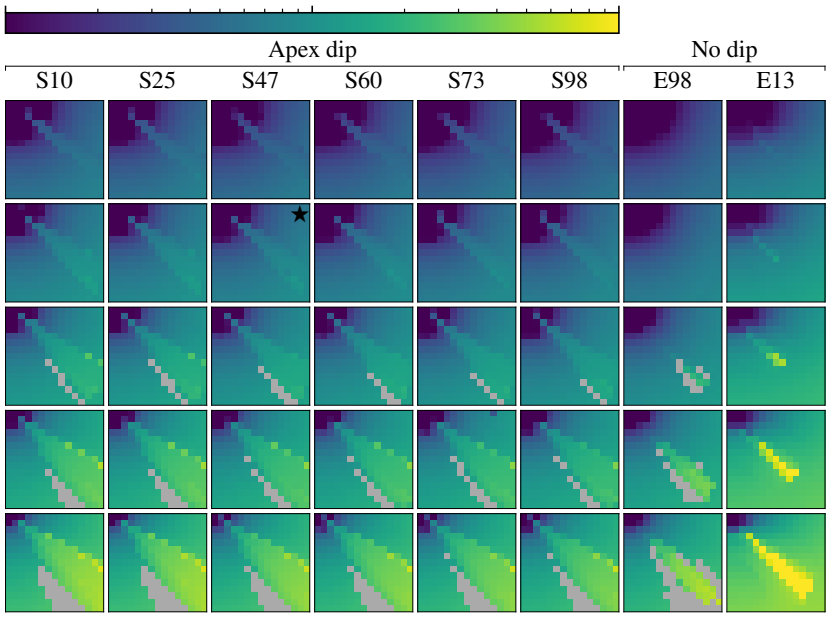

(d) Event classification

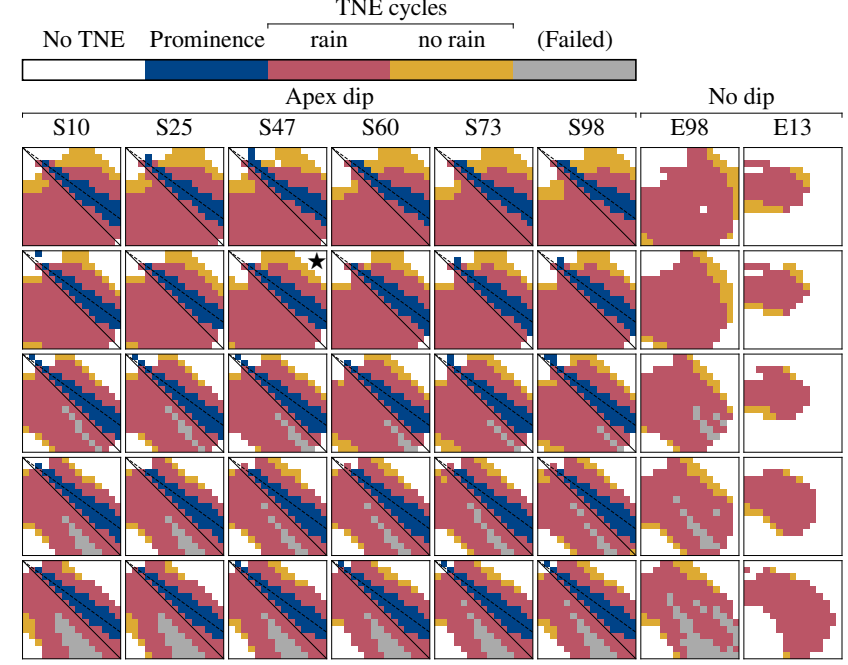

Fig. 4. Characteristic quantities for the 9000 simulations of the rain bow. (a) Maximum temperature, (b) maximum electron number density, and (c) average velocity, computed around the loop apex $\left(z>0.9 z_{\max }\right)$ during the second half of each simulation ( $t>36 \mathrm{~h}$ ). (d) Event classification: no TNE behaviour ( $\square$ ), formation of a prominence-like structure $(\square)$, TNE cycles with $(\square)$ and without $(\square)$ coronal rain, and simulations that failed because of numerical issues $(\square)$. Each sub-figure contains 40 squares, corresponding to the eight line geometries (columns), and the five values of $H_{1} / H_{0}$ (rows). Each square contains 225 simulations for the different values of $\lambda_{1}$ ( $y$-axis) and $\lambda_{2}$ ( $x$-axis), ranging from $1 \%$ of the loop length (top left) to $20 \%$ of the loop length (bottom right). The solid lines on (d) correspond to $\lambda_{1}=\lambda_{2}$. The dashed lines correspond to $\lambda_{1}=0.73 \lambda_{2}$. The square marked with a star $(\star)$ corresponds to the 225 simulations shown in Fig. 3a.

(in blue in Fig. 4d) form for relatively symmetric heating functions, with scale heights close to the line $\lambda_{1}=0.73 \lambda_{2}$. In contrast, TNE cycles with coronal rain (in red) occur for more asymmetric heating functions, and cycles without coronal rain (in yellow) for even more asymmetric functions. In a small fraction of the parameter space, the loop can reach a steady state, and thus shows no TNE behaviour (in white). Finally, some simulations fail prematurely because of numerical issues (in grey) and, therefore, we could not determine their behaviour. Theses numerical issues occur when massive condensations fall through the chromospheric layer and reach a boundary of the computation domain (see Sect. 3.1). Such condensations form for relatively strong and symmetric heating functions. The strong heating causes important evaporation, while its symmetry allows for the condensations to gain significant mass before falling.

Figure 4a shows the maximum temperature reached around the loop apex during the second half of each simulation. Simulations with TNE cycles reach higher temperatures than the neigh- bouring simulations without cycles, which is consistent with the results of Froment et al. (2018). In addition, loops with a larger area expansion reach slightly lower maximum temperatures (the maximum temperatures reached with the S98 geometry are approximately $10 \%$ lower than those reached with the S10 geometry). This effect has been explained for static loops by Vesecky et al. (1979): a larger area expansion increases the upwards thermal conduction, which is, in turn, compensated for by larger radiative losses in the corona. Because the radiative losses are a decreasing function of the temperature in the corona, this results in lower coronal temperatures. However, the temperature variation remains small compared to the cross-sectional area variation. The maximum temperature also increases when the heating rate $H_{1} / H_{0}$ increases.

Figure $4 \mathrm{~b}$ shows the maximum electron number density reached around the loop apex during the second half of the simulations. The maximum electron density is higher in simulations close to the diagonal $\lambda_{1}=\lambda_{2}$. However, there is no clear-cut 
relationship between the simulations that produce prominencelike structures, and adjacent simulations that produce TNE cycles with coronal rain. The maximum electron density increases with the heating rate $H_{1} / H_{0}$ (as expected from loop scaling laws), and slightly decreases with the loop area expansion. Similarly to the temperature, the density variation is small compared to the cross-sectional area variation (approximately a $25 \%$ difference between S10 and S98). In some simulations, the plasma $\beta$ (i.e. the ratio of gas and magnetic pressures) is greater than one, which breaks the assumption that the magnetic field does not evolve. This occurs for simulations with a large heating rate $\left(H_{1} / H_{0}\right)$ and large scale heights $\left(\lambda_{1}\right.$ and $\left.\lambda_{2}\right)$. In the most extreme case, the plasma $\beta$ locally reaches 500 . However, such deviations only occur locally, when complete condensations are formed. As a result, their effect is limited to the local dynamics and morphology of the coronal rain or prominence. Thus, we do not expect them to have an effect on whether condensations are formed in the first place. In addition, the actual plasma $\beta$ is likely to be lower than the aforementioned value because the intensity of the extrapolated magnetic field is probably underestimated (see discussion in Appendix B). Finally, while beyond the assumptions of the simulation, a high plasma $\beta$ is consistent with the observations. It would allow the massive condensations to deform the magnetic field and form the observed apex dip. The gradual formation of the apex dip would also explain why coronal rain is only seen to linger at the end of the sequence observed by Auchère et al. (2018).

Figure $4 \mathrm{c}$ shows the average velocity around the loop apex during the second half of the simulations. For low heating rates $H_{1} / H_{0}$, simulations with prominence-like structures and neighbouring simulations forming TNE cycles display similar apex velocities. In this case, the average velocities are of the order $+5 \mathrm{~km} \mathrm{~s}^{-1}$ when $\lambda_{1}>0.6 \lambda_{2}$ (i.e. simulations for which $\lambda_{1}>$ $\lambda_{2}$, plus simulations with prominence-like structures), and of the order of $-5 \mathrm{~km} \mathrm{~s}^{-1}$ otherwise. For higher heating rates, simulations with prominence-like structures have higher average apex velocities than neighbouring simulations with TNE cycles, reaching values of $\sim 8 \mathrm{~km} \mathrm{~s}^{-1}$. In simulations that form a prominencelike structure, upflows develop in both legs of the loop and feed material into the prominence, while the velocity in the apex dip is zero. The north edge of the dip (where velocities are positive) has a higher altitude (see Fig. 2), and thus contributes more than the south edge to the velocity averages shown in Fig. 4c. This explains why the averaged velocities around the apex are positive in simulations that form prominence-like structures.

\subsubsection{Extrapolated geometries}

We now present the results of the simulations performed with the geometries E13 and E98, obtained through magnetic field extrapolation. The goal of these simulations is to explore how the presence of the dip at the apex of the stereoscopically reconstructed plasma affects the evolution of the loops. Geometry E98 is similar to geometry S98, but with no apex dip: it has a length of $629 \mathrm{Mm}$ (635 Mm for S98), a relative area expansion of 98 (identical to that of S98), and a similar altitude profile in the loop legs. Geometry E13 has an area expansion of 13 (thus comparable to that of S10), but a much shorter length (465.54 Mm).

The characteristic values and event classification of the simulations using these geometries are shown in Fig. 4. Simulations of E98 mainly differ from those of S98 in the fact that they do not form prominence-like structures. This is the direct result of the fact that E98 has no apex dip. As a result, all condensations fall along the loop legs, resulting in TNE cycles. Apart from this, simulations of E98 and S98 have overall similar characteristics, including the period of the TNE cycles. This suggests that the presence of an apex dip leads to the formation of prominencelike structures when the heating is relatively symmetric, but it does not change the characteristics of TNE cycles otherwise. On the other hand, simulations of E13 have very different characteristics. This is primarily explained by the fact that geometry E13 is significantly shorter than the other geometries.

\subsection{Reproducing the observations of the rain bow}

Additionally, we used the simulations to determine the heating parameters and the cross-sectional area expansion of the rain bow. To that end, we searched for a simulation that uses the extrapolated geometry (S10-S98), has TNE cycles with abundant formation of coronal rain, and has the closest period to that of the observed pulsations $(6.7 \mathrm{~h})$. The simulation which best matches these criteria has a relative area expansion of 60 (geometry S60), and a symmetric, highly stratified heating $\left(\lambda_{1}=\lambda_{2}=\right.$ $15 \mathrm{Mm}=0.024 L)$ with a high heating rate $\left(H_{1}=20480 H_{0}=\right.$ $\left.307 \mu \mathrm{W} \mathrm{m}^{-3}\right)$. It displays TNE cycles with a period of $8 \mathrm{~h}$ and abundant formation of coronal rain. The simulations using the extrapolated geometries S10 to S60 and the aforementioned heating parameters $\left(\lambda_{1}, \lambda_{2}\right.$, and $\left.H_{1}\right)$ all have a similar behaviour and period. However, simulations that use other heating parameters display periods of at least $10 \mathrm{~h}$. Hence, the heating parameters are well constrained, while the loop area expansion is not.

In Fig. 5, we show the evolution of the temperature (a), electron number density (b), and velocity (c) as a function of the position along the loop and time during two pulsation periods. The temperature and density profiles show that a large condensation periodically forms at the top of the south leg (around $s=375 \mathrm{Mm}$, which is about $25 \mathrm{Mm}$ away from the south edge of the apex dip). This condensed plasma is then evacuated along the same footpoint, thus resulting in coronal rain. While the condensation falls, a zone of lower density and higher velocity forms at the opposite footpoint. When the condensation reaches the chromosphere, a smaller condensation bounces back up, but it does not reach the loop apex. A perturbation travelling at the sound speed is also created, which reaches the opposite footpoint.

In Fig. 5d, we show the evolution of the temperature, electron number density, and velocity at the location where the condensation forms (near the apex for $370 \mathrm{Mm}<s<380 \mathrm{Mm}$ ), and in the leg along which it falls $(500 \mathrm{Mm}<s<510 \mathrm{Mm})$. Near the apex, the cycle starts with a temperature of $0.8 \mathrm{MK}$ and a density of $10^{14} \mathrm{~m}^{-3}$. The plasma starts to condense at the apex, with the temperature decreasing to $0.1 \mathrm{MK}$ while the density increases to $7 \times 10^{14} \mathrm{~m}^{-3}$. The condensed plasma then starts to fall along the loop (the apex velocity reaches $25 \mathrm{~km} \mathrm{~s}^{-1}$ ). As it falls along the loop, the plasma continues to condense and its velocity increases. In the loop leg, the temperature decreases to $0.05 \mathrm{MK}$, the density increases to $1 \times 10^{16} \mathrm{~m}^{-3}$, and the velocity increases to $100 \mathrm{~km} \mathrm{~s}^{-1}$.

The condensation starts falling with a constant acceleration of about $5 \mathrm{~m} \mathrm{~s}^{-2}$ along the loop. The acceleration then increases in the loop leg (for $s>420 \mathrm{Mm}$, where the loop is steeper), and it reaches a maximum value of $40 \mathrm{~m} \mathrm{~s}^{-2}$. Auchère et al. (2018) measured coronal rain acceleration in the rain bow loop and reported values ranging from $5 \mathrm{~m} \mathrm{~s}^{-2}$ to $10 \mathrm{~m} \mathrm{~s}^{-2}$ on the plane of the $\mathrm{sky}^{2}$. While the simulation features a larger acceleration in

2 The angle between the loop and the plane of the sky (POS) about $10^{\circ}$ in this section of the loop. Thus, the accelerations projected onto the POS is about the same as along the loop. 
(a) Temperature

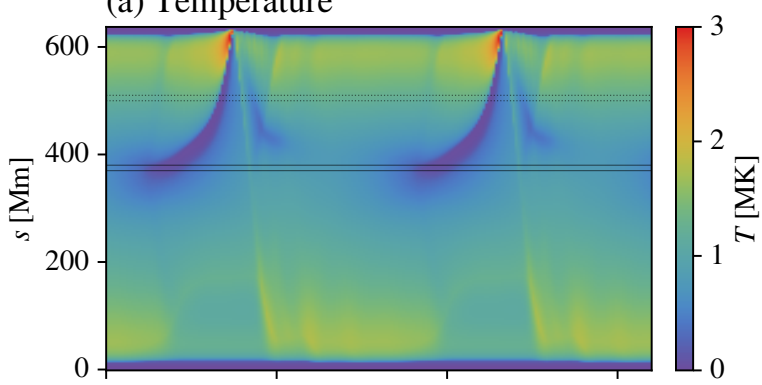

(b) Electron number density

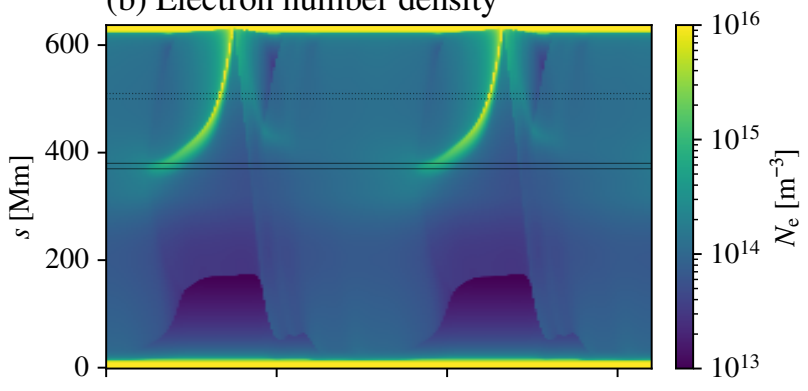

(c) Velocity

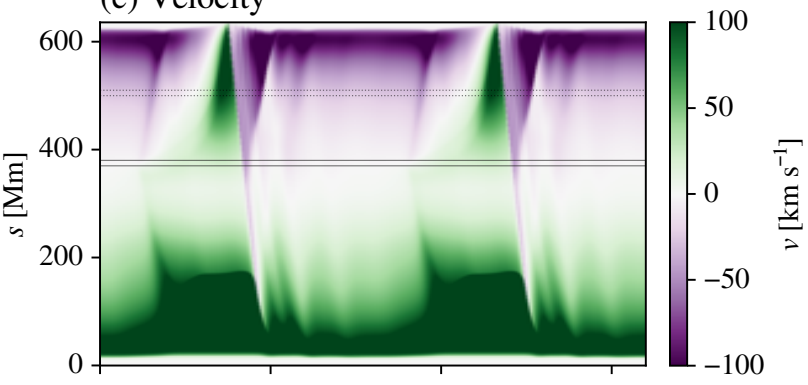

(d) Averaged apex and leg quantities

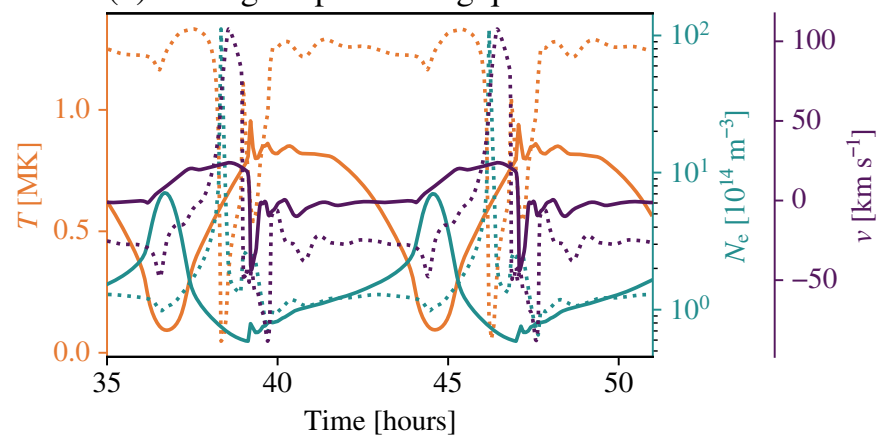

Fig. 5. Simulation that reproduces the observations of the rain bow best, obtained with geometry S60, $H_{1} / H_{0}=20480$, and $\lambda_{1}=\lambda_{2}=15 \mathrm{Mm}$. (a) Temperature, (b) electron number density, and (c) velocity, as a function of the position along the loop and time. (d) Temperature (orange), electron number density (blue), and velocity (purple) averaged close to the apex $(370 \mathrm{Mm}<s<380 \mathrm{Mm}$, solid lines) and in the leg (500 Mm $<s<510 \mathrm{Mm}$, dashed lines). The apex and leg regions are also shown in sub-figures (a), (b), and (c). The figure shows times from 35 to $51 \mathrm{~h}$ out of the $72 \mathrm{~h}$ of the simulation. The temporal evolution of the temperature, electron number density, and velocity along the loop can be seen in the online movie.

the lower part of the loop, it is overall consistent with the observations. In particular, these values are about five times lower than the gravitational acceleration projected along the loop (e.g. $26 \mathrm{~m} \mathrm{~s}^{-2}$ where the condensation starts to fall, and $235 \mathrm{~m} \mathrm{~s}^{-2}$ when it reaches maximum acceleration). The observed and simulated acceleration profiles are typical of coronal rain, which is known to have velocities lower than the free-fall speed (see e.g., Schrijver 2001; De Groof et al. 2005; Antolin et al. 2010; Vashalomidze et al. 2015). This happens because coronal rain is slowed down by forces that oppose gravity, such as the pressure gradient (Oliver et al. 2014) or drag (in multidimensional simulations, Martínez-Gómez et al. 2020).

The period of the TNE cycles in this simulation is $20 \%$ longer than the one observed in the rain bow. However, this is the simulation using stereoscopically reconstructed geometries with the smallest period. Johnston et al. (2019) show that the period of TNE pulsations can increase if the background heating $\left(H_{0}\right.$ in our case) is too high. They report a period increase for background heating values above $10^{3} \mathrm{nW} \mathrm{m}^{-3}$. Although we did not include different values of $H_{0}$ in our parametric study, the background heating values that we used $\left(<55 \mathrm{nW} \mathrm{m}^{-3}\right)$ should thus be low enough to not modify the TNE periods.

To better compare to the observations of Auchère et al. (2018), we computed synthetic intensities for this simulation. The intensities were computed using the SDO/AIA temperature response functions from Chianti 8 (Dere et al. 1997; Del Zanna et al. 2015), and the method described in Froment et al. (2017, Sect. 3.2.1). In Fig. 6a, we show the evolution of the intensity in the 171,193 , and $335 \AA$ bands of AIA, as a function of the position along the loop and time. In Fig. 6b, we show the evolution of the intensity integrated close to the apex (between 370 and $380 \mathrm{Mm}$ ) for the six coronal channels of $\mathrm{AIA}^{3}$. The temperature-response function of these bands is shown in Fig. 6c. The intensity first peaks in the $171 \AA$ band (maximum temperature response at $0.9 \mathrm{MK}$ ), which is consistent with the fact that the loop reaches a maximum temperature of $0.8 \mathrm{MK}$ during the cycle. The intensity then peaks in the other bands in the following order: $94 \AA, 131 \AA$, $193 \AA, 211 \AA$, and $335 \AA$. These peaks, except $94 \AA$, correspond to local maximums of each band's temperature response function below $0.8 \mathrm{MK}$, peaking in order of decreasing temperature. This behaviour is consistent with the numerous reports that coronal loops (undergoing TNE or not) are generally observed in their cooling phase (Warren et al. 2002; Winebarger et al. 2003; Winebarger \& Warren 2005; Ugarte-Urra et al. 2006, 2009; Mulu-Moore et al. 2011; Viall \& Klimchuk 2011, 2012; Froment et al. 2015). However, the bands peak in a different order in the observations of the rain bow, with $193 \AA, 94 \AA$, $211 \AA$, and $335 \AA$ peaking before $171 \AA$ (Auchère et al. 2018). While this order is also consistent with loops observed in their cooling phase, it indicates that the maximum temperature observed in the rain bow ranges between 1 and $1.5 \mathrm{MK}$, thus higher than in our simulation. This temperature difference would explain why the simulated AIA bands peak in a different order than in the observations.

The simulation that best matches the observed behaviour of the rain bow reproduces its main observed characteristics: the development of TNE cycles with abundant formation of coronal rain. While it does not precisely reproduce some quantitative details of the observation (the pulsation period and the maximum temperature), the qualitative behaviour is similar. This allows us to conclude that a highly stratified, symmetric heating function is required in order to produce the periodic coronal rain observed in the rain bow. We can also conclude that these loops must have a large cross-sectional area expansion.

\footnotetext{
3 We note that the choice of the integration region does not significantly influence the order in which different bands peak.
} 
(a) Intensities along the loop

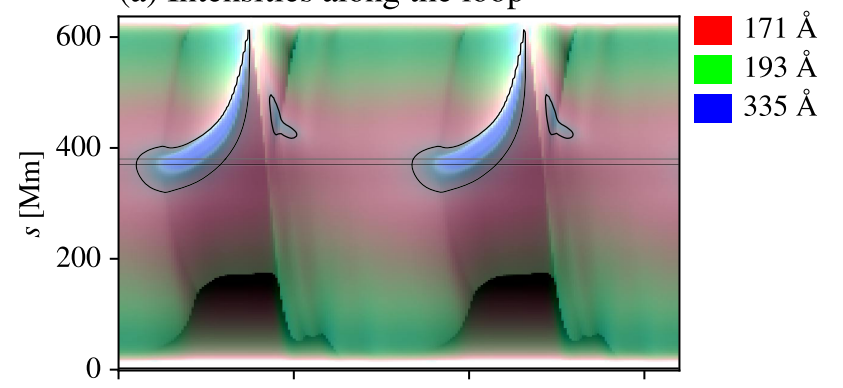

(b) Integrated apex intensities

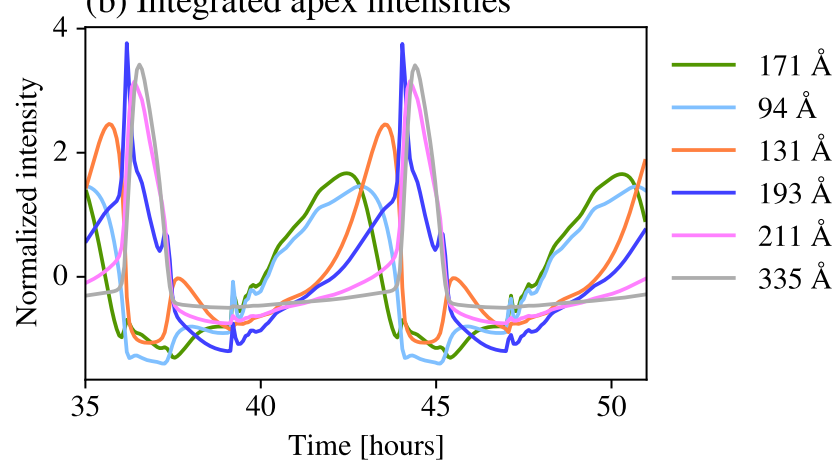

(c) AIA temperature response

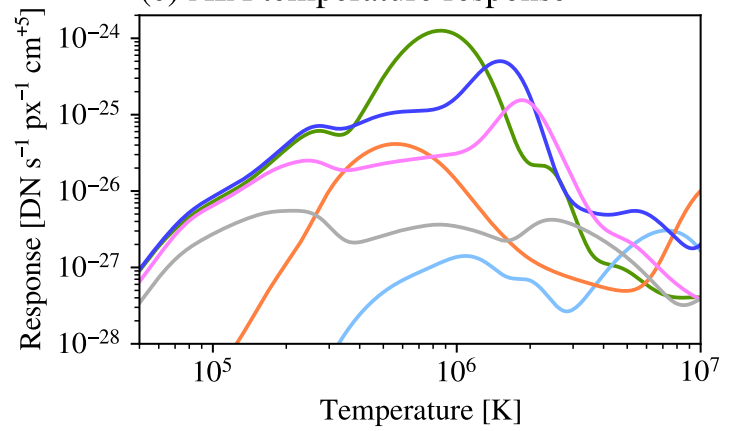

Fig. 6. Synthetic intensities for the simulation that reproduces the observations of the rain bow best. (a) Intensity in the $171 \AA$ (red), $193 \AA$ (green), and $335 \AA$ (blue) bands of SDO/AIA as a function of the position along the loop and time. The black contour shows regions where the temperature is lower than $0.5 \mathrm{MK}$. (b) Intensities in the six coronal channels of AIA, integrated close to the apex, $(370 \mathrm{Mm}<s<380 \mathrm{Mm})$. Each light curve was normalised by subtracting its average and dividing by its standard deviation. (c) Temperature-response function of the SDO/AIA channels shown in panel (b).

\section{The role of asymmetries in the formation of coronal rain}

\subsection{Simulation statistics}

To get an overview of the different kind of events obtained in the simulations of the rain bow, we counted the number of simulations showing each kind of behaviour: TNE cycles with and without rain, the formation of a prominence-like structure, relaxation to a steady-state (i.e. no TNE), and failed simulations. The counts are given in Table 2. In this table, we also include the fraction of the explored parameter space corresponding to each type of event. Because these values depend on the choice of the parameter space, they do not constitute a precise measurement of the expected event type. Rather, they offer a synthetic view of the trends visible in Fig. 4.
We first consider the 6750 simulations performed with the six stereoscopically reconstructed geometries (S10 to S98). These simulations undergo TNE in a large fraction of the explored parameter space $(78.5 \%)$. They are split in three groups: cycles with coronal rain $(69.0 \%$ of simulations with TNE, or $86.3 \%$ of simulations with TNE cycles), formation of a prominence-like structure $(20.0 \%)$, and cycles without rain $(11.0 \%)$. In $18.2 \%$ of the parameter space, simulations relax to a steady state, thus showing no TNE behaviour. Finally, 3.3\% of the simulations fail because of the numerical issues discussed in Sect. 3.1.

The 1125 simulations performed with the extrapolated geometry E98 have relatively similar statistics. A slightly smaller fraction of the parameter space experiences TNE $(67.7 \%)$, and no simulations form a prominence-like structure. However, $87.3 \%$ of TNE cycles produce coronal rain, which is very close to the value obtained for geometries S10 to S98 $(86.3 \%)$. In addition, more simulations, albeit still a small fraction, fail because of numerical issues $(5.6 \%)$.

Finally, the 1125 simulations using the extrapolated geometry E13 have rather different statistics. A much smaller fraction of the parameter space experiences TNE (34.7\%). Nonetheless, a similar fraction of cycles produce coronal rain $(88.2 \%)$. None of the simulations using this geometry fail because of numerical issues. These differences are explained by the fact that the length and altitude profile of geometry E13 strongly differ from those of the other geometries (see Sect. 2).

Overall, a large fraction of the parameter space that we explored experiences TNE. Periodic coronal rain is easily formed in the rain bow, with on average $86 \%$ of TNE cycles forming coronal rain for the parameters we explored.

\subsection{Interplay between the asymmetry of the loop and of the heating}

We have shown that TNE cycles with coronal rain occur preferentially in the simulations of the rain bow. Indeed, they are the most common behaviour, found in $51 \%$ of all simulations and in $86 \%$ of simulations with TNE cycles. The simulated behaviour is consistent with the observation of abundant periodic coronal rain showers (the 'monsoon') in the rain bow loop (Auchère et al. 2018).

While the rain bow loop has characteristics similar to those of the loop studied by Froment et al. (2015), the latter had little or no rain. In order to understand this different behaviour, we compared the simulations of the rain bow to the simulations performed for three different geometries by Froment et al. (2018). The authors used the same simulation set-up and heating function as in the current paper. They considered a semi-circular loop geometry with a length of $367 \mathrm{Mm}$ (loop A), a very asymmetric loop obtained from magnetic field extrapolation (loop B, matching the observations for which no rain was detected), and a relatively symmetric loop also obtained from extrapolation (loop C). Loop B corresponds to a loop bundle in which TNE cycles with little to no coronal rain had been previously identified (Froment et al. 2015). Its eastern leg (at $s=0$ ) has a high inclination, resulting in a very small projected gravity in the first $10 \%$ of the loop length (Froment et al. 2018, Fig. 3). Loop C is a loop in which no TNE behaviour was observed. The legs of this loop are relatively symmetric. The classification of the events Froment et al. (2018) obtained for these loops is reproduced in Fig. 7 for easier comparison with our simulations of the rain bow (Fig. 4d).

The simulations performed by Froment et al. (2018) for the two symmetric geometries preferentially produce coronal rain (84.6\% TNE of cycles in loop A and $99.4 \%$ of cycles in loop C 
Table 2. Event type counts in all simulations of the rain bow.

\begin{tabular}{|c|c|c|c|c|c|c|c|c|c|}
\hline & \multicolumn{3}{|c|}{ S10 to S98 } & \multicolumn{3}{|c|}{ E98 } & \multicolumn{3}{|c|}{ E13 } \\
\hline & Count & Frac. tot. & Frac. TNE & Count & Frac. tot. & Frac. TNE & Count & Frac. tot. & Frac. TNE \\
\hline \multicolumn{10}{|l|}{ TNE behaviours: } \\
\hline Prominence $\mathbf{\square}$ & 1063 & $15.7 \%$ & $20.0 \%$ & - & - & - & - & - & - \\
\hline Cycle with rain $\square$ & 3658 & $54.2 \%$ & $69.0 \%$ & 665 & $59.1 \%$ & $87.3 \%$ & 344 & $30.6 \%$ & $88.2 \%$ \\
\hline $\begin{array}{l}\text { Cycle without rain } \\
\text { (all) }\end{array}$ & $\begin{array}{c}582 \\
(5303)\end{array}$ & $\begin{array}{c}8.6 \% \\
(78.5 \%)\end{array}$ & $11.0 \%$ & $\begin{array}{c}97 \\
(762)\end{array}$ & $\begin{array}{c}8.6 \% \\
(67.7 \%)\end{array}$ & $12.7 \%$ & $\begin{array}{c}46 \\
(390)\end{array}$ & $\begin{array}{c}4.1 \% \\
(34.7 \%)\end{array}$ & $11.8 \%$ \\
\hline No TNE $\square$ & 1227 & $18.2 \%$ & & 300 & $26.7 \%$ & & 735 & $65.3 \%$ & \\
\hline Failed simulations & 220 & $3.3 \%$ & & 63 & $5.6 \%$ & & - & - & \\
\hline
\end{tabular}

Notes. For each geometry, we give the number of simulations showing each event type (Count column), the fraction compared to the total number of simulations using the geometry (Frac. tot. column), and the fraction compared to the number of simulations showing TNE (Frac. TNE column).

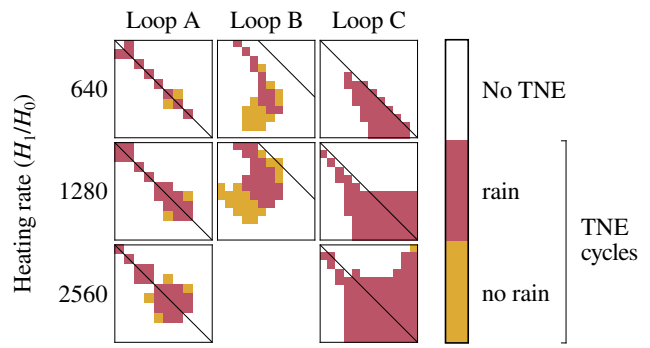

Fig. 7. Event classification for the simulations performed by Froment et al. (2018), shown with a similar layout as Fig. 4d. Loop A is a semicircular with a length of $367 \mathrm{Mm}$, heated with ten values of $\lambda_{1}$ and $\lambda_{2}$, both ranging from 2 to $11 \%$ of the loop length. Loop B is a highly asymmetric loop with a length of $367 \mathrm{Mm}$, heated with 12 values of $\lambda_{1}$ and $\lambda_{2}$, ranging from 7 to $18 \%$ and from 2 to $13 \%$ of the loop length, respectively. Loop $\mathrm{C}$ is a moderately asymmetric loop with a length of $139 \mathrm{Mm}$, heated with 12 values of $\lambda_{1}$ and $\lambda_{2}$, both ranging from 4 to $15 \%$ of the loop length. The diagonal lines in each square correspond to $\lambda_{1}=\lambda_{2}$.

produce rain). However, less rain forms in the very asymmetric loop B (only $50.7 \%$ of the cycles produce rain).

This comparison between the simulations of the rain bow and the simulations of Froment et al. (2018) suggests that the geometry of the loop has, on its own, a significant effect on the formation of coronal rain during TNE cycles. In a relatively symmetric loop, coronal rain forms for a wider range of heating functions. On the other hand, cycles without rain occur more easily in very asymmetric loops ${ }^{4}$. However, the heating function also plays a key role in the formation of rain. In the corona, a given loop is only subject to one of the many explored heating functions. The development of TNE thus results from a specific match between the loop geometry and the heating function, as noted by Froment et al. (2018). Our simulations of the rain bow support this conclusion and further suggest that symmetric loops more frequently produce cycles with coronal rain, while asymmetric loops lead to more cycles without rain. In the case of the rain bow, cycles with no rain only occur in a small volume of the parameter space. This makes such cycles very unlikely to occur in a real coronal loop, as it would require the heating and geometry to be stable for several days. On the other hand, cycles with rain could be produced even with vary-

\footnotetext{
4 We verified that these conclusions are unchanged if we only consider the simulations by Froment et al. (2018) and the ones of the rain bow that have overlapping heating parameters.
}

ing heating or geometry, provided that the variations are slow enough.

Overall, coronal rain is formed if the condensing plasma cools down to chromospheric temperatures before being evacuated from the loop. In this case, the condensed plasma then falls along the loop as coronal rain. On the contrary, no rain is formed if the condensations are destabilised earlier and fall while still at coronal temperatures. In relatively symmetric loops (e.g., the rain bow), condensations can remain at the loop apex longer for a wide variety of heating functions. Only very asymmetric heating functions lead to cycles with no rain. As a result, such loops preferentially form cycles with rain. In asymmetric loops (e.g., loop B of Froment et al. 2018), condensations can only remain at the apex if an oppositely asymmetric heating creates upflows that compensate for the loop's asymmetry. In such loops, rain is overall less likely to occur as it can only form under specific heating conditions.

Our simulations of the rain bow and the simulations of Froment et al. (2018) are also consistent with the qualitative predictions of the analytical formulas of Klimchuk \& Luna (2019), which provide conditions for TNE (with or without rain) to occur in coronal loops. We compared the simulations to the overall predictions of these formulas, but did not perform a quantitative comparison. Their first condition (Klimchuk \& Luna 2019, Eq. (13)) is that the heating must be concentrated at low altitudes. This is the case in all simulations. The second condition (Klimchuk \& Luna 2019, Eq. (34)) is that the product of the asymmetries in the heating and the cross-sectional area must not be too important. In the case of the rain bow, the loop shape and area expansion are relatively symmetric. As a result, a wider variety of heating functions, including highly asymmetric ones, may lead to TNE. On the other hand, loop B of Froment et al. (2018) has a very asymmetric shape and area expansion. This imposes a larger constraint on the heating function: In order to have TNE, the heating must also be asymmetric in order to compensate for the geometric asymmetry of the loop.

\subsection{Other parameters that may influence the formation of coronal rain}

In the current paper, we explore different loop geometries and heating parameters, and their influence on the development of TNE in coronal loops. However, other factors may influence the evolution of plasma in a loop. In particular, we did not consider any variation in the heating or the geometry over time. Variations in the heating over short time scales (i.e. less than the loop 
cooling time) have been considered in a few studies of TNE. Antolin et al. (2010) and Susino et al. (2010) have shown that complete plasma condensations can occur in impulsively heated loops. Winebarger et al. (2018) and Johnston et al. (2019) have studied the influence of impulsive heating on some characteristics of TNE cycles. Most of these studies consider heating functions with a constant waiting time between the pulses. It would also be worth considering stochastic heating functions, as these better describe nanoflare heating mechanisms in the solar corona. Stochastic heating functions have been considered in several simulations of coronal loops (e.g., in OD by Cargill 2014; Cargill et al. 2015; Barnes et al. 2016, and in 1D by Cargill et al. 2015; Bradshaw \& Viall 2016), but only in a few simulations of TNE cycles (Antolin et al. 2010; Antolin 2020). Including such impulsive stochastic heating functions in large-scale parametric studies is an essential next step to understand the relationship between heating and TNE cycles.

As noted in previous studies of TNE cycles (Antolin et al. 2010; Auchère et al. 2018; Froment et al. 2018), the heating parameters and loop geometry are also likely to vary over longer time scales (i.e. longer than the loop cooling time), thus resulting in a variation in the TNE conditions over time. This could result in non-periodic TNE cycles, cycles only lasting for a few periods, or the intermittent production of coronal rain from one cycle to another. Understanding the range of behaviours that can stem from such long-term variations would require exploring a much larger parameter space than considered in the current study. In the case of the rain bow, one could take the evolution of the loop geometry over time into account. Doing so would require the reconstruction of the loop geometry at several time intervals, which would be an even greater challenge than the reconstruction presented in Sect. 2.

The conclusions presented in this paper are based on simulations of realistic loop geometries, which were derived from observations. The symmetric and asymmetric loops result in clearly distinct TNE evolutions. However, it is unclear whether the formation of coronal rain is constrained by asymmetries of specific geometric features (such as the cross-sectional area or the slope of the loop legs) or if it results from any geometric asymmetry. We note that the asymmetric loop B of Froment et al. (2018) has a unique geometry, with an asymmetric cross-sectional area and one very inclined leg. This leg is almost horizontal over its first $30 \mathrm{Mm}$, and it partly lays in the chromosphere. We also note that the loops considered by Froment et al. (2018) and in the current paper have very different area expansion profiles (Fig. 2c). However, this is unlikely to have a significant influence compared to the loop asymmetry because loops $\mathrm{A}$ and $\mathrm{C}$ also preferentially produce coronal rain, despite having different expansion profiles than the rain bow loops. In order to understand whether the formation of coronal rain is influenced by asymmetries in specific parts of the loop, it would be interesting to realise simulations for a wider variety of loop geometries. The use of synthetic geometries would allow one to better control the different geometric features.

\section{Summary}

In this paper, we present 1D hydrodynamic simulations of the rain bow, a periodic coronal rain event observed by Auchère et al. (2018). This event produces abundant coronal rain showers, which strongly contrasts with previous observations of TNE cycles that did not show evidence of coronal rain (Froment et al. 2015).
To understand which parameters influence the formation of coronal rain during TNE cycles, we performed 9000 simulations of the rain bow, exploring different heating parameters (volumetric heating rate and asymmetry) and variants of the geometry (shape and area expansion of the loop). To perform these simulations, we reconstructed the 3D geometry of the loop.

These simulations display the three behaviours that can result from TNE in coronal loops: evaporation and condensation cycles with coronal rain, cycles without coronal rain, or the formation of a prominence-like structure. We thus show that prominences and TNE cycles can develop in similar conditions (same geometry and similar heating parameters). This constitutes a new result for understanding TNE in the solar corona.

By searching for the simulation that best reproduces the observed characteristics of the rain bow, we estimated the heating conditions of this event. The rain bow event results from an intense and symmetric heating localised near the footpoints of the loop (heating scale-height of $15 \mathrm{Mm}$ in both legs). Furthermore, a small variation in the heating function results in the formation of a prominence-like structure. This would also be consistent with the onset prominence formation reported by Auchère et al. (2018).

Finally, we used the large parametric study to explore the relationship between the geometry of the loop, the asymmetry of the heating, and the formation of coronal rain during TNE cycles. We compared the simulations of the rain bow (symmetric loop) with another set of simulations performed by Froment et al. (2018) (two symmetric loops and one asymmetric loop). We conclude that coronal rain is overall more likely to occur in relatively symmetric loops. In such loops, rain forms for a wide range of heating functions. In asymmetric loops, however, rain can only form if the heating precisely compensates for the geometric asymmetry, so that condensations can remain in the corona long enough to reach chromospheric temperatures. When the heating does not compensate for the loop asymmetry, condensations are evacuated from the loop before they can cool down to chromospheric temperatures, resulting in cycles with no coronal rain.

Froment et al. (2018) already noted that TNE requires a specific match between the loop geometry and the heating conditions. The simulations of the rain bow suggest that a symmetric loop allows for a looser match between the geometry and the heating. Both behaviours are consistent with the qualitative predictions of the formulas of Klimchuk \& Luna (2019).

Acknowledgements. We thank Peter Cargill for his in depth referee review, which significantly improved the manuscript. G. P. has received funding from the European Research Council (ERC) under the European Union's Horizon 2020 research and innovation program (grant agreement No. 724326). We acknowledge support from the International Space Science Institute (ISSI), Bern, Switzerland to the International Team 401 "Observed Multi-Scale Variability of Coronal Loops as a Probe of Coronal Heating". AIA and HMI data are courtesy of NASA/SDO and the AIA and HMI science teams. EUVI data are courtesy of the STEREO SECCHI team. This work used data provided by the MEDOC data and operations centre (CNES/CNRS/Univ. Paris-Sud), medoc . ias . u-psud . fr. CHIANTI is a collaborative project involving George Mason University, the University of Michigan (USA), University of Cambridge (UK) and NASA Goddard Space Flight Center (USA). Software: Astropy (Astropy Collaboration 2013, 2018), ChiantiPy (Dere 2013), JHelioviewer (Müller et al. 2017), SolarSoft (Freeland \& Handy 2012)

\section{References}

Antiochos, S. K., \& Klimchuk, J. A. 1991, ApJ, 378, 372

Antiochos, S. K., MacNeice, P. J., Spicer, D. S., \& Klimchuk, J. A. 1999, ApJ, 512, 985

Antiochos, S. K., MacNeice, P. J., \& Spicer, D. S. 2000, ApJ, 536, 494 Antolin, P. 2020, PPCF, 62, 014016 
G. Pelouze et al.: Asymmetries and coronal rain in TNE cycles

Antolin, P., \& Rouppe van der Voort, L. 2012, ApJ, 745, 152

Antolin, P., Shibata, K., \& Vissers, G. 2010, ApJ, 716, 154

Antolin, P., Vissers, G., \& Rouppe van der Voort, L. 2012, Sol. Phys., 280, 457

Aschwanden, M. J., Newmark, J. S., Delaboudinière, J.-P., et al. 1999, ApJ, 515, 842

Astropy Collaboration (Robitaille, T. P., et al.) 2013, A\&A, 558, A33

Astropy Collaboration (Price-Whelan, A. M., et al.) 2018, AJ, 156, 123

Auchère, F., Bocchialini, K., Solomon, J., \& Tison, E. 2014, A\&A, 563, A8

Auchère, F., Froment, C., Soubrié, E., et al. 2018, ApJ, 853, 176

Barnes, W. T., Cargill, P. J., \& Bradshaw, S. J. 2016, ApJ, 833, 217

Boerner, P., Edwards, C., Lemen, J., et al. 2012, Sol. Phys., 275, 41

Bradshaw, S. J., \& Viall, N. M. 2016, ApJ, 821, 63

Cargill, P. J. 2014, ApJ, 784, 49

Cargill, P. J., Warren, H. P., \& Bradshaw, S. J. 2015, RSPTA, 373, 20140260

Craig, I. J. D., \& Schulkes, R. M. S. M. 1985, ApJ, 296, 710

Cranmer, S. R., \& Winebarger, A. R. 2019, ARA\&A, 57, 157

De Groof, A., Berghmans, D., van Driel-Gesztelyi, L., \& Poedts, S. 2004, A\&A 415,1141

De Groof, A., Bastiaensen, C., Müller, D. a. N., Berghmans, D., \& Poedts, S. 2005, A\&A, 443, 319

Delaboudinière, J.-P., Artzner, G. E., Brunaud, J., et al. 1995, Sol. Phys., 162, 291

Del Zanna, G., Dere, K. P., Young, P. R., Landi, E., \& Mason, H. E. 2015, A\&A, 582, A56

Dere, K. 2013, Astrophysics Source Code Library [record ascl:1308.017]

Dere, K. P., Landi, E., Mason, H. E., Monsignori Fossi, B. C., \& Young, P. R. 1997, A\&AS, 125,

Dere, K. P., Landi, E., Young, P. R., et al. 2009, A\&A, 498, 915

Domingo, V., Fleck, B., \& Poland, A. I. 1995, Sol. Phys., 162, 1

Downs, C., Lionello, R., Mikić, Z., Linker, J. A., \& Velli, M. 2016, ApJ, 832, 180

Driesman, A., Hynes, S., \& Cancro, G. 2008, Space Sci. Rev., 136, 17

Dudík, J., Dzifčáková, E., \& Cirtain, J. W. 2014, ApJ, 796, 20

Feldman, U. 1992, Phys. Scr., 46, 202

Feldman, U., Mandelbaum, P., Seely, J. F., Doschek, G. A., \& Gursky, H. 1992, ApJS, 81, 387

Foukal, P. 1978, ApJ, 223, 1046

Freeland, S. L., \& Handy, B. N. 2012, Astrophysics Source Code Library [record ascl:1208.013]

Froment, C. 2016, PhD Thesis, Université Paris-Saclay, Université Paris-Sud, Institut d'Astrophysique Spatiale, Orsay, France

Froment, C., Auchère, F., Bocchialini, K., et al. 2015, ApJ, 807, 158

Froment, C., Auchère, F., Aulanier, G., et al. 2017, ApJ, 835, 272

Froment, C., Auchère, F., Mikić, Z., et al. 2018, ApJ, 855, 52

Froment, C., Antolin, P., Henriques, V. M. J., Kohutova, P., \& Rouppe van der Voort, L. H. M. 2020, A\&A, 633, A11

Grevesse, N., \& Sauval, A. J. 1998, Space Sci. Rev., 85, 161

Howard, R. A., Moses, J. D., Vourlidas, A., et al. 2008, Space Sci. Rev., 136, 67

Johnston, C. D., Cargill, P. J., Antolin, P., et al. 2019, A\&A, 625, A149

Kaiser, M. L., Kucera, T. A., Davila, J. M., et al. 2008, Space Sci. Rev., 136, 5

Karpen, J. T., \& Antiochos, S. K. 2008, ApJ, 676, 658

Karpen, J. T., Antiochos, S. K., Hohensee, M., Klimchuk, J. A., \& MacNeice, P. J. 2001, ApJ, 553, L85

Karpen, J. T., Tanner, S. E. M., Antiochos, S. K., \& DeVore, C. R. 2005, ApJ, 635,1319

Karpen, J. T., Antiochos, S. K., \& Klimchuk, J. A. 2006, ApJ, 637, 531

Kawaguchi, I. 1970, PASJ, 22, 405

Klimchuk, J. A. 2019, Sol. Phys., 294, 173
Klimchuk, J. A., \& Luna, M. 2019, ApJ, 884, 68

Koechlin, L., Dettwiller, L., Audejean, M., Valais, M., \& López Ariste, A. 2019, A\&A, 631, A55

Kuin, N. P. M., \& Martens, P. C. H. 1982, A\&A, 108, L1

Landi, E., Feldman, U., \& Dere, K. P. 2002, ApJS, 139, 281

Landi, E., Young, P. R., Dere, K. P., Del Zanna, G., \& Mason, H. E. 2013, ApJ, 763,86

Lemen, J. R., Title, A. M., Akin, D. J., et al. 2012, Sol. Phys., 275, 17

Leroy, J.-L. 1972, Sol. Phys., 25, 413

Lionello, R., Linker, J. A., \& Mikić, Z. 2009, ApJ, 690, 902

Martens, P. C. H., \& Kuin, N. P. M. 1983, A\&A, 123, 216

Martínez-Gómez, D., Oliver, R., Khomenko, E., \& Collados, M. 2020, A\&A, 634, A36

McWhirter, R. W. P., Thonemann, P. C., \& Wilson, R. 1975, A\&A, 40, 63

Mikić, Z., Lionello, R., Mok, Y., Linker, J. A., \& Winebarger, A. R. 2013, ApJ, 773, 94

Mok, Y., Drake, J. F., Schnack, D. D., \& van Hoven, G. 1990, ApJ, 359, 228

Mok, Y., Mikić, Z., Lionello, R., \& Linker, J. A. 2005, ApJ, 621, 1098

Mok, Y., Mikić, Z., Lionello, R., Downs, C., \& Linker, J. A. 2016, ApJ, 817, 15

Müller, D. a. N., Hansteen, V. H., \& Peter, H. 2003, A\&A, 411, 605

Müller, D. a. N., Peter, H., \& Hansteen, V. H. 2004, A\&A, 424, 289

Müller, D. A. N., De Groof, A., Hansteen, V. H., \& Peter, H. 2005, A\&A, 436, 1067

Müller, D., Nicula, B., Felix, S., et al. 2017, A\&A, 606, A10

Mulu-Moore, F. M., Winebarger, A. R., Warren, H. P., \& Aschwanden, M. J. 2011, ApJ, 733, 59

Oliver, R., Soler, R., Terradas, J., Zaqarashvili, T. V., \& Khodachenko, M. L. 2014, ApJ, 784, 21

O'Shea, E., Banerjee, D., \& Doyle, J. G. 2007, A\&A, 475, L25

Pelouze, G., Auchère, F., Bocchialini, K., et al. 2020, A\&A, 634, A54

Pesnell, W. D., Thompson, B. J., \& Chamberlin, P. C. 2012, Sol. Phys., 275, 3

Pottasch, S. R. 1965, Bull. Atron. Inst. Neth., 18, 7

Priest, E. R., Hood, A. W., \& Anzer, U. 1989, ApJ, 344, 1010

Régnier, S. 2013, Sol. Phys., 288, 481

Scherrer, P. H., Schou, J., Bush, R. I., et al. 2012, Sol. Phys., 275, 207

Schrijver, C. J. 2001, Sol. Phys., 198, 325

Scullion, E., Rouppe van der Voort, L., Antolin, P., et al. 2016, ApJ, 833, 184

Spitzer, L. 1962, Physics of Fully Ionized Gases, 2nd edn. (New York: Interscience)

Susino, R., Lanzafame, A. C., Lanza, A. F., \& Spadaro, D. 2010, ApJ, 709, 499

Ugarte-Urra, I., Winebarger, A. R., \& Warren, H. P. 2006, ApJ, 643, 1245

Ugarte-Urra, I., Warren, H. P., \& Brooks, D. H. 2009, ApJ, 695, 642

Vashalomidze, Z., Kukhianidze, V., Zaqarashvili, T. V., et al. 2015, A\&A, 577, A136

Vesecky, J. F., Antiochos, S. K., \& Underwood, J. H. 1979, ApJ, 233, 987

Viall, N. M., \& Klimchuk, J. A. 2011, ApJ, 738, 24

Viall, N. M., \& Klimchuk, J. A. 2012, ApJ, 753, 35

Warren, H. P., Winebarger, A. R., \& Hamilton, P. S. 2002, ApJ, 579, L41

Wiegelmann, T., Thalmann, J. K., \& Solanki, S. K. 2014, A\&ARv, 22, 78

Winebarger, A. R., \& Warren, H. P. 2005, ApJ, 626, 543

Winebarger, A. R., Warren, H. P., \& Seaton, D. B. 2003, ApJ, 593, 1164

Winebarger, A. R., Lionello, R., Downs, C., Mikić, Z., \& Linker, J. 2018, ApJ, 865,111

Wuelser, J.-P., Lemen, J. R., Tarbell, T. D., et al. 2004, Telesc. Instrum. Sol. Astrophys., 5171, 111

Xia, C., \& Keppens, R. 2016, ApJ, 823, 22

Xia, C., Chen, P. F., Keppens, R., \& van Marle, A. J. 2011, ApJ, 737, 27

Xia, C., Keppens, R., Antolin, P., \& Porth, O. 2014, ApJ, 792, L38 


\section{Appendix A: Stereoscopic reconstruction}

We reconstructed the shape of the rain bow loop (Auchère et al. 2018) using stereoscopic observations of SDO/AIA and STEREO/SECCHI/EUVI. In this appendix, we summarise the method and the results pertinent to the present work. More details will be given in a forthcoming companion paper.

The rain bow event was observed at the limb by AIA for 2.5 days starting from 2012 July 23, 00:00 UT. During this period, the STEREO B probe had a separation of $115^{\circ}$ with Earth, and it observed the event on-disk. The event was not visible from STEREO A. The EUVI data consist of images in the $171 \AA$ band with a 120 min cadence, and in the $304 \AA$ band with a cadence of $2.5 \mathrm{~min}$ for the first $200 \mathrm{~min}$ and $10 \mathrm{~min}$ afterwards. The temperature response function of the $171 \AA$ band peaks at $0.9 \mathrm{MK}$, which allows the observation of plasma at coronal temperatures. The $304 \AA$ band peaks at $0.09 \mathrm{MK}$, allowing the observation of coronal rain. During the same time period, AIA images are available in these two bands with a cadence of $12 \mathrm{~s}$.

The coronal rain is easily observed at the limb in the AIA $304 \AA$ images. We used a combination of intensity thresholding and manual clean-up to identify the regions of the images which contain coronal rain. The coronal rain is harder to see on-disk in the EUVI $304 \AA$ images. To better detect it, we divided the intensity of each image by the average of the 20 previous and 20 following images. The resulting intensity maps were then used to manually select the regions where coronal rain is observed.

For each pair of $304 \AA$ images from SDO and STEREO B, we thus obtained two binary masks, containing the regions in which coronal rain is observed. In order to reconstruct the longest possible fraction of the loop, we selected a pair of masks in which the rain occupies a large fraction of the loop seen in the $171 \AA$ images. The selected images were captured on 2012 July 25, 03:06 UT, near the end of the observing sequence. We then used the two masks to reconstruct the volume occupied by the coronal rain. To that end, we traced the lines of sight of each rain pixel in the masks and computed the intersection between the lines of sight from SDO and from STEREO B. Such a stereoscopic reconstruction technique assumes that both instruments observe the same opaque object. However, that is not entirely the case here: Only the dense core of the rain is visible on-disk in absorption from STEREO B, while a larger volume of the rain is seen off-limb in emission from SDO. Additionally, ambiguities occur when rain is detected in adjacent loop threads. When both instruments observe rain in two adjacent threads, four distinct threads are present in the volume reconstruction. But only two of these threads are real. We resolved such ambiguities manually by ensuring the continuity of threads along the loop and in time. The loop footpoints were inferred manually as they are partially hidden behind the limb in the AIA image, and they are difficult to see in the EUVI images in which the rain forms very thin strands. To that end, we extended the volume occupied by the coronal rain down to the solar surface, while trying to preserve the overall shape of the loop. This affects the lowest $5 \mathrm{Mm}$ of the northern leg and the lowest $20 \mathrm{Mm}$ of the southern leg. Finally, we fitted a spline to the remaining voxels. This yielded an approximation for the shape of the rain bow loop. The resulting 3D shape is shown in Fig. 1. The altitude and projected gravity and are shown in Fig. 2 (a) and (b).

The stereoscopic reconstruction reveals a dip near the apex of the loop. While this feature is unusual for coronal loops, it is consistent with some prominence models (e.g., Priest et al. 1989). Such a dip is consistent with the observations of Auchère et al. (2018), who report that the condensations linger at the apex before falling, and they suggest a possible link with prominence formation. This dip appears to be a robust result from the reconstruction, and we are rather confident that it is not an artefact. The dip is located at the centre of the loop $(s=319 \mathrm{Mm})$ and at the altitude $z=118 \mathrm{Mm}$. The southern edge of the dip is located very close to the dip, both in regards to the position along the loop (32 Mm away, at $s=351 \mathrm{Mm}$ ) and in altitude $(2 \mathrm{Mm}$ higher, at $z=120 \mathrm{Mm})$. The northern edge is further from the dip along the loop (87 Mm away, at $s=232 \mathrm{Mm}$ ) and in altitude $(13 \mathrm{Mm}$ higher, at $z=131 \mathrm{Mm})$. On this northern side, the altitude reaches $120 \mathrm{Mm}$ (the height of the south edge) at $s=298 \mathrm{Mm}$. Overall, the dip has a depth of $2 \mathrm{Mm}$ and a width of $53 \mathrm{Mm}$.

\section{Appendix B: Magnetic field extrapolation}

To determine the area expansion of the rain bow loop (Auchère et al. 2018), we performed a potential magnetic field extrapolation from a synoptic line of sight magnetogram of SDO/HMI. The synoptic magnetogram has a resolution of $0.5^{\circ}$ pixel $^{-1}$ in Carrington coordinates. The magnetic field at each longitude was measured when the corresponding meridian was as the centre of the disk as seen from SDO (i.e. approximately from Earth). For the Carrington longitudes between $0^{\circ}$ and $191^{\circ}$, we used magnetograms recorded at the rotation before the observation of the rain bow (CR2125), that is about 20 days before. For longitudes between $191^{\circ}$ and $360^{\circ}$, we used magnetograms from the following rotation (CR2126), recorded a few days after the observation of the rain bow. The boundary between the magnetograms from both rotations is thus located between the footpoints of the stereoscopically reconstructed shape $S$ (at Carrington longitudes $185^{\circ}$ and $207^{\circ}$, respectively). The resulting magnetogram is shown in Fig. B.1. This composite magnetogram contains the polarities required to obtain extrapolated lines with a shape resembling that of the rain bow: a magnetic quadrupole at the east of the loops (marked by grey circles in Fig. B.1), which explains their inclination. This quadrupole is not present when only using data from rotation CR2125 (because the south-west negative polarity had not emerged yet), nor when only using data from rotation CR2126 (because the north-west positive polarity has decayed too much).

We then performed a potential extrapolation, where it is assumed that the magnetic field satisfies the condition $\boldsymbol{\nabla} \times \boldsymbol{B}=\mathbf{0}$ (see, e.g., Régnier 2013; Wiegelmann et al. 2014). We selected four magnetic field lines extrapolated from this magnetogram: E08, E13, E25, and E98. The properties of these extrapolated lines are summarised in Table B.1. Lines E13 and E98 are shown in Figs. 1, 2, and B.1. Similarly to the stereoscopically reconstructed loop shape (Appendix A), the extrapolated lines are nonplanar and inclined towards the east of the solar sphere.

The footpoint magnetic field of these loops ranges from 5 to $60 \mathrm{G}$, which is somewhat smaller than typical active region values (see e.g., Aschwanden et al. 1999 who reported a range of 20-230 $\mathrm{G}$ for a selection of 30 active region loops). This can be explained by the low resolution of the magnetogram, which reduces and spreads out the magnetic field intensity. Line E98 has an area expansion of 98 , which is higher than typical active region values (Dudík et al. 2014 report a maximum value of 80 in a given active region). Overall, potential magnetic field extrapolation from a low resolution magnetogram only gives a very coarse estimation of the magnetic field, especially in active regions. However, the main goal of these extrapolations is to estimate the relative loop cross-sectional area (a more precise estimation of the loop shape has already been obtained from the 


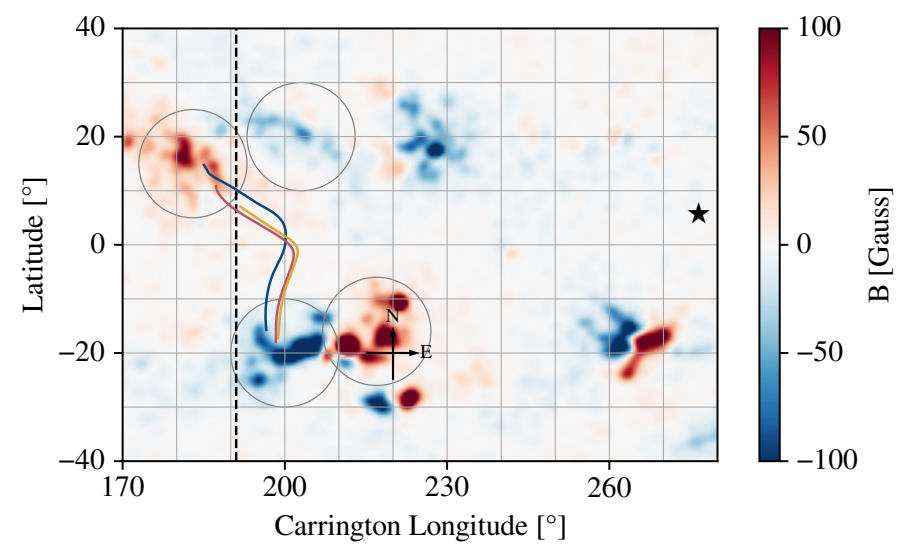

Fig. B.1. Magnetogram used for the magnetic field extrapolation. The vertical dashed line at the longitude of $191^{\circ}$ corresponds to the limit between the synoptic magnetograms from rotations CR2125 (left) and CR2126 (right). The quadrupolar structure mentioned in the text is marked by grey circles. The black star indicates the solar disk centre as seen from Earth at the time for which geometry $\mathrm{S}$ was reconstructed (2012 July 25, 03:06 UT). The loop geometries, the heliographic grid, and direction cross shown in Fig. 1 were reproduced for easier comparison.

stereoscopic reconstruction). Nonetheless, because of the uncertainty of this extrapolation method, we explored different crosssectional area profiles.

We thus generated six relative loop area profiles from the extrapolated lines (E08, E13, E25, and E98). We combined these six area profiles with the shape from the stereoscopic
Table B.1. Properties of the extrapolated field lines used to generate area expansion profiles for the stereoscopic loop shape $\mathrm{S}$. The length $L$, the magnetic field at the north footpoint $B(0)$, at the south footpoint $B(L)$, and the minimum value close to the apex $B_{\min }$ are provided.

\begin{tabular}{ccccc}
\hline \hline Line & $L[\mathrm{Mm}]$ & $B(0)[\mathrm{G}]$ & $B_{\min }[\mathrm{G}]$ & $B(L)[\mathrm{G}]$ \\
\hline E08 & 430.63 & 5.06 & 0.60 & 44.78 \\
E13 & 465.54 & 8.59 & 0.65 & 47.02 \\
E25 & 492.24 & 23.45 & 0.95 & 48.37 \\
E98 & 628.86 & 41.92 & 0.43 & 62.68 \\
\hline
\end{tabular}

reconstruction $\mathrm{S}$. The resulting geometries share the same shape, but have different area profiles, with maximum values ranging from 10 to 98.4. These new geometries are labelled with their maximum relative area expansion: S10, S25, S47, S60, S73, and S98. Because the lines have different lengths, we scaled each area expansion profile to the loop length. The new area expansion profiles were generated from the extrapolated area expansion profiles using the following relations:

$$
\begin{aligned}
& A_{S 10}\left(s / L_{S 10}\right)=\left[A_{E 08}\left(s / L_{E 08}\right)+A_{E 13}\left(s / L_{E 13}\right)\right] / 2, \\
& A_{S 25}\left(s / L_{S 25}\right)=A_{E 25}\left(s / L_{E 25}\right), \\
& A_{S 47}\left(s / L_{S 47}\right)=\left[2 A_{E 25}\left(s / L_{E 25}\right)+A_{E 98}\left(s / L_{E 98}\right)\right] / 3, \\
& A_{S 60}\left(s / L_{S 60}\right)=\left[A_{E 25}\left(s / L_{E 25}\right)+A_{E 98}\left(s / L_{E 98}\right)\right] / 2, \\
& A_{S 73}\left(s / L_{S 73}\right)=\left[A_{E 25}\left(s / L_{E 25}\right)+2 A_{E 98}\left(s / L_{E 98}\right)\right] / 3, \\
& A_{S 98}\left(s / L_{S 98}\right)=A_{E 98}\left(s / L_{E 98}\right) .
\end{aligned}
$$

These cross-sectional area profiles are shown in Fig. 2 (c). 